L. Ingber, "Statistical mechanics of combat and extensions," in Toward a Science of Command, Control, and Communications, ed. by C. Jones (American Institute of Aeronautics and Astronautics Washington, D.C., 1993, pp. 117-149)

\title{
Statistical Mechanics of Combat and Extensions
}

\author{
Lester Ingber ${ }^{*}$ \\ Lester Ingber Research, P.O. Box 857, McLean, VA 22101
}

Copyright (C) by the American Institute of Aeronautics and Astronautics, Inc. All rights reserved.

*President, EMail: ingber@alumni.caltech.edu 


\section{Introduction: $\mathbf{C}^{2}$ in Training and Computer Models}

\section{A. Necessity of Comparing Computer Models to Exercise Data}

This project addresses the utility of establishing a mathematical approach to compare exercise data to large scale computer models whose underlying microscopic interactions among men and machines are driven by the natural laws of physics. In this study, the focus is to compare the Janus(T) wargame to National Training Center (NTC) data, since both systems then take into account human interactions.

It also should be noted that "large scale" here refers to battalion level. (Army systems scale by factors of 3-5, from company to battalion to brigade to division to corps to army.) If these battalion level computer models can be favorably compared, and if consistency can be achieved between the hierarchy of large scale battalion level, larger scale corps level, and largest scale theater level computer models, then these higher echelon computer models also can be favorably compared. This could only enhance the value of training on these higher echelon computer models. ${ }^{1}$

The requirement of depending more and more on combat computer models (including simulations and wargames) has been brought into sharper focus because of many circumstances, e.g.: (1) the nonexistence of ample data from previous wars and training operations, (2) the rapidly shortening time scale on which tactical decisions must be made, (3) the rapidly increasing scale at which men and machines are to be deployed, (4) the increasing awareness of new scenarios that are fundamentally different from historical experiences, (5) and the rapidly increasing expense of conducting training exercises.

Furthermore, such computer models could be used to augment training. We now spend several million dollars to cycle each battalion through NTC. The training of these commanders could be greatly enhanced if inexpensive pre and post training wargames were provided that statistically replicate their training missions. Even, or rather especially, for the development of such training aids, proper analysis and modeling is required to quantitatively demonstrate that the computer models are good statistical representations of the training mission.

However, the level of acceptance of computer models in major military battle-management and procurement decisions appears to be similar to the level of acceptance of computer simulations in physics in the 1960s. In physics, prior to the 1960s, theory and experiment formed a close bond to serve to understand nature. In the 1960s, academicians were fascinated with evolving computer technology, but very few people seriously accepted results from computer simulations as being on a par with good theory and good experiment. Now, of course, the situation is quite different. The requirement of understanding truly complex systems has placed computer simulation, together with theory and experiment, as an equal leg of a tripod of techniques used to investigate physical nature.

The requirements necessary to bring combat computer models to their needed level of importance are fairly obvious. To have confidence in computer-model data, responsible decision makers must be convinced that computer models model reality, not metaphors of reality, models of models, or models of models of models, etc. Many people feel that not much progress has been made in the last decade ${ }^{2,3}$ with regard to this issue, despite a general awareness of the problem.

If a reasonable confidence level in computer models of large scale combat scenarios could be obtained, there are several immediate payoffs to be gained. More objective data could be presented for procurement decisions, e.g., provided by sensitivity analyses of sets of computer models differing in specific weapons characteristics. To give proper weight to these differing characteristics, their influence within the global context of full combat scenarios would be tested.

\section{B. Need for Data}

With the present development of high quality simulators (essentially computer simulations coupled with hardware to simulate close to actual machinery and communication) to augment training in the field, e.g., SIMNET, it should become increasingly important to "validate" the simulator system to some reality, even if only exercise reality. For example, several hundred simulators are likely to be driven in the context of tens of thousands of units being simulated by software similar to present-day computer simulations. In fact, many people see the necessity of integrating simulations and simulators to properly augment training. Thus, the semi-automated forces (SAF) driving the simulators must be validated. We believe we offer here the appropriate methodology to carry out this program. 
As we will see, proper high quality validation requires "high quality" data, recorded in "timely" fashion. The proper time scale required for validation of battalion- to brigade level combat appears to be on the order of minutes. High quality typically means accurate "killer victim" scoreboards (KVS) as a function of time. This timely and high quality data is currently only available from such exercise arenas as NTC, certainly not from records of actual combat. However, qualifying NTC data is laborious, and in these days of diminishing research and development funding, it might soon become a vanishing art form. This does not diminish the requirement for gathering more high quality data for analysis, if analysis is to properly serve training; nor does it excuse the expenditure of billions of dollars on hardware without spending at least millions on validation of software driving this machinery.

\section{Large Scale $\mathbf{C}^{2}$ and Need for Mathematical Modeling}

Modeling phenomena is as much a cornerstone of 20th century science as is collection of empirical

data. ${ }^{4}$ In practically all fields of science, mathematical models of the real world become tested by fitting some parameters to empirical data. Since systems in the real world are often nonlinear and stochastic, it is not surprising that often this fitting process must involve fitting statistical, nonlinear, functional (algebraic) forms to data. They are nonlinear because typically their alternative outputs can be complicated functions of their inputs and feedback. They are stochastic because typically they have many constituents that are generally treated as aggregate entities, and/or the specification of these entities requires statistical judgment about past or future performance.

As in other fields of science, in the context of modeling combat, reductionist doctrine is simply inadequate to fully understand large scale systems. For example, a threshold is quickly reached at a level of any large system, be it physical, biological or social, when a "language" shift is required for effective command and control. A high level commander cannot use a grease board to track individual units, albeit he might periodically sample his units, but he/she must rather look at the overall systematics, e.g., aggregated measures of force (MOF) or effectiveness (MOE), attrition, resupply, etc. At this level we properly require command and control $\left(\mathrm{C}^{2}\right)$, rather than "supra-battle-management" from commanders. At this level we denote the system as large scale.

This issue of utilizing MOFs and MOEs, e.g., starting at approximately battalion level of combat, is relevant to computer models as well actual combat. Merely aggregating data to form MOFs or MOEs does not determine if results from one mission (combat or computer-model scenario) are comparable to another mission. For example, small differences in tempo or in spatial distribution of forward line of own troops (FLOT), or forward edge of the battle area (FEBA), may cause tables of numbers to appear quite different.

Mathematical models of aggregated data should be expected to uncover "mechanisms" of combat, e.g., line firing or area firing in simple Lanchester theory. More complex missions plausibly will contain more subtle mechanisms as well as weighted contributions of more basic mechanisms, e.g., quantification of dynamic "force," "stress," "strain," "momentum," "energy," etc., as is possible with this approach. Using this as hindsight, in some systems it may then be possible to specify a figure of merit, some simple set of numbers to capsulate the influence of these mechanisms.

It must be emphasized that this approach requires an evolution of knowledge. This project is developing models suitable to describe the statistical nature of selected force-on-force battalion and brigade scenarios. It is expected that the accumulation of models of many types of scenarios will lead to a better fundamental understanding of combat with direct operational applications.

\section{Models Versus Reality}

It must be stated that there are still many problems faced by all computer models of combat which must be solved before they can be accepted as models of reality. For example, a very basic problem exists in the quality of acquisition algorithms, i.e., how to construct an algorithm that realistically portrays human attention (preattentive as well as selective) and perception, under various combat and weather conditions, night vs day, etc. The influence of attention and perception on complex physical ${ }^{5-7}$ and mental $\operatorname{tasks}^{8,9}$ has received considerable attention by the author. Currently, the best combat computer models treat acquisition as serial and logical processes, whereas the human brain acquires data by parallel and associative processes. Therefore, the inclusion of human players in multiple runs of similar scenarios is essential, if a probabilistic mathematical model is to be developed to model exercise data such as that 
obtained from NTC.

Now, line of sight (LOS) algorithms seem to be the most costly time factor in running Janus(T) computer models. Even if more realistic acquisition algorithms are developed, they must be tailored to the needs of real time computer models if they are to be used in wargaming and in training.

\section{E. Outline of Paper}

Section II describes the present day empirical approximate of the real world suitable for gathering data, e.g., NTC. We must model for reasons given above. Good physical theory always must interact with the real world, and so we must see what observed data is available.

Section III demonstrates that the natural evolution of mathematical methodology often starts out in a strikingly simple fashion describing some region of appropriate reality. Given the form that data is collected at NTC and from Janus(T), i.e., KVSs, a natural formal structure is intuited for this beginning mathematical description.

However, as is true for many physical systems, simple equations describing one patch of reality often can require quite abstract and sophisticated transformations and algorithms to faithfully calculate other empirical observables in some other region of reality. In fact, the degree to which theory can extrapolate or at least interpolate from one region of reality to another is the practical scientific method of testing theories. Therein lies much of the utility of theories, to present patterns of information to human minds, more significant than enumeration of tables of statistics and empirical data. Complex reality therefore, not surprisingly, requires complex theoretical structures, and such complex structures require good quality data to fit a theory to one region and to test the theory in other regions. In this case, nonlinear stochastic combat leads to nonlinear stochastic equations, requiring state-of-the-art methodologies much more advanced than available even only a decade ago.

Section IV presents the numerical algorithms required to faithfully carry our the calculations intended by the mathematics derived in Sec. III. The numerical algorithms required likewise are state-ofthe-art. Without these numerical techniques, the mathematical formalism of Sec. III would have to be drastically undercut or shelved, leading to abuses of modeling of actual NTC and Janus data, more typical (and then excusable) of pioneering work done decades ago without such formalism and numerical techniques.

Section V gives a short outline of the relevance of our work to finding mathematical "chaos" in battalion level combat. The presence of chaos simply is not supported by the facts, tentative as they are because of sparse data.

Section VI describes an ambitious but reasonable approach to more explicitly model human factors in combat, using techniques developed for describing command and control of teleoperated vehicles.

Section VII describes extension of our statistical mechanics of combat (SMC) methodology to other scenarios. This work was done with several officer students of the author.

\section{Janus Computer Simulation of National Training Center}

\section{A. Individual Performance}

Clearly individual performance is extremely important in combat, ${ }^{10}$ ranging in scale from battle management of the commander, to battle leadership of sub-commanders, to the degree of participation of individual units, to the more subtle degradation of units performing critical tasks.

Our analyses of NTC data concludes that the quality of data collected to date is not sufficient to accurately statistically judge individual performance across these scales. However, we do believe that this data is sufficient to analyze battle management and perhaps battle leadership at the company or platoon level, in some cases reflecting the influence of a human commander.

It is important to recognize and emphasize the requirement of improving data collection at NTC, to permit complementary analyses of human factors at finer scales than our statistical approach permits.

Therefore, as understood from experience in simulating physics systems, many trajectories of the "same" stochastic system must be aggregated before a sensible resolution of averages and fluctuations can be ascertained. Given two scenarios that differ in one parameter, and given a sufficient number of trajectories of each scenario, then the sensitivity to changes of a "reasonable" algebraic function to this parameter can offer some analytic input into decisions involving the use of this parameter in combat scenarios. 
NTC is the best source of such data, albeit it is sparse.

\section{B. Description of National Training Center}

The U.S. Army National Training Center (NTC) is located at Fort Irwin, just outside Barstow, California. As of 1989, there have been about 1/4 million soldiers in 80 brigade rotations at NTC, at the level of two battalion task forces (typically about 3500 soldiers and a battalion of 15 attack helicopters), which train against two opposing force (OPFOR) battalions resident at NTC. NTC comprises about $2500 \mathrm{~km}^{2}$, but the current battlefield scenarios range over about $5 \mathrm{~km}$ linear spread, with a maximum lethality range of about $3 \mathrm{~km}$. NTC is gearing up for full brigade level exercises.

Observer-controllers (OC) are present at various levels of command down to about platoon level. A rotation will have three force-on-force missions and one live-fire mission. OPFOR platoon and company level personnel are trained as US Army soldiers; higher commanders practice Soviet doctrine and tactics. An OPFOR force typically has $\sim 100$ red armored personnel carriers (BMPs) and $\sim 40$ red tanks (T72s).

The primary purpose of data collection during an NTC mission is to patch together an after action review (AAR) within a few hours after completion of a mission, giving feedback to a commander who typically must lead another mission soon afterward. Data from the field, i.e., multiple integrated laser engagement system (MILES) devices, audio communications, OCs, and stationary and mobile video cameras, is sent via relay stations back to a central command center where this all can be recorded, correlated and abstracted for the AAR. Within a couple of weeks afterwards, a written review is sent to commanders, as part of their NTC take home package. It now costs about $4 \times 10^{6}$ dollars per NTC rotation, 1 million of which goes for this computer support.

There are 460 MILES transponders available for tanks for each battle. The B units have transponders, but most do not have transmitters to enable complete pairings of kills targets to be made. (New MILES devices being implemented have transmitters which code their system identification, thereby greatly increasing the number or recordings of pairings.) Thus, MILESs without transmitters cannot be tracked. Man packs with B units enable these men to be tracked, but one man pack can represent an aggregate of as many as five people.

B units send data to A stations (was 48, though 68 can be accommodated), then collected by two C stations atop mountains, and sent through cables to central VAXs forming a core instrumentation system (CIS). There is a present limitation of 400 nodes in computer history for video tracking (but 500 nodes can be kept on tape). Therefore, about 200 blue and 200 OPFOR units are tracked.

By varying the laser intensity and focusing parameters, a maximum laser-beam spread is achieved at the nominal range specified by the Army. A much narrower beam can reach as far as the maximum range. Focusing and attenuation properties of the laser beam makes these nominal and maximum ranges quite sharp, with resolution supposedly considerably less than several hundred meters under ideal environmental conditions. For example, a weapon might send out a code of 8 words (spaced apart by ns), 2 of which must register on a target to trigger the Monte Carlo routine to calculate a probability of kill (PK). Attenuation of the beam past its preset range means that it rapidly becomes unlikely that 2 words will survive to reach the target.

With increasing demands to make training more realistic, the MILES devices need to be upgraded. For example, degradation of the laser beam under conditions of moderate to heavy smoke and dust might be at least partially offset by sending fewer words per message. New sensor abilities to encode specific shooters will also greatly aid data collection.

It should be understood that present training problems at NTC, e.g., training commanders-especially at Company level - to handle synchronization of more than three tasks, misuse of weapons systems, etc., overshadow any problems inherent in the MILES systems. We repeatedly have expressed this view for well over a year, after going to NTC several times; but only at a meeting at Carlisle Barracks, PA, on May 17, 1989, when various school commanders briefed Gen. Maxwell Thurman, TRADOC Commander, was this view broadly accepted.

Therefore, to the degree possible in this project, our wargaming efforts strive to place commanders under these constraints of current interest, e.g., under requirements to synchronize the timing of the movement or repositioning of forces, request for supporting fires (artillery, air strike, etc.), initiation of fires into target zones, the shifting of supporting fires, the execution of local counter-attacks, etc. 


\section{Qualification Process}

Missing unit movements and initial force structures were completed in the NTC database, often making "educated guesses" by combining information on the CIS tapes and the written portion of the take-home package.

This project effectively could not have proceeded if we had not been able to automate transfers of data between different databases and computer operating systems. One of the author's students, Bowman, ${ }^{11}$ wrote a thesis on the management of the many information-processing tasks associated with this project. He has coordinated and integrated data from NTC, Training and Doctrine Command (TRADOC) Analysis Command (TRAC) at White Sands Missile Range, New Mexico (TRAC-WSMR) and at Monterey, California (TRAC-MTRY) for Janus(T) wargaming at TRAC-MTRY, and for use at at Lawrence Livermore National Laboratory (LLNL) Division B, and for Janus(T) and NTC modeling.

\section{Description of Janus(T)}

Janus(T) is an interactive, two-sided, closed, stochastic, ground combat computer simulation. As discussed below, we have expanded Janus(T) to include air and naval combat, in several projects with the author's previous thesis students at the Naval Postgraduate School (NPS).

Interactive refers to the the fact that military analysts (players and controllers) make key complex decisions during the simulation, and directly react to key actions of the simulated combat forces. Twosided (hence the name Janus of the Greek two-headed god) means that there are two opposing forces simultaneously being directed by two set of players. Closed means that the disposition of the enemy force is not completely known to the friendly forces. Stochastic means that certain events, e.g., the result of a weapon being fired or the impact of an artillery volley, occur according to laws of chance [random number generators and tables of probabilities of detection (PD), acquisition (PA), hit (PH), kill (PK), etc.]. The principle modeling focus is on those military systems that participate in maneuver and artillery operations. In addition to conventional direct fire and artillery operations, Janus(T) models precision guided munitions, minefield employment and breaching, heat stress casualties, suppression, etc.

Throughout the development of Janus(T), and its Janus precursor at Lawrence Livermore National Laboratory, extensive efforts have been made to make the model "user friendly," thereby enabling us to bring in commanders with combat experience, but with little computer experience, to be effective wargamers. There is now a new version, Janus(A), bringing together the strengths of these predecessors.

\section{Mathematical Formalism}

\section{A. Model Development}

Consider a scenario taken from our NTC study: two red systems, red T-72 tanks $(R T)$ and red armored personnel carriers $(R B M P)$, and three blue systems, blue M1A1 and M60 tanks (BT), blue armored personnel carriers $(B A P C)$, and blue tube-launched optically-tracked wire-guided missiles $(B T O W)$, where $R T$ specifies the number of red tanks at a given time $t$, etc. Consider the kills suffered by $B T, \Delta B T$, e.g., within a time epoch $\Delta t \approx 5$ min

$$
\Delta B T / \Delta t \equiv \dot{B T}=x_{R T}^{B T} R T+y_{R T}^{B T} R T B T+x_{R B M P}^{B T} R B M P+y_{R B M P}^{B T} R B M P B T
$$

Here, the $x$ terms represent attrition owing to point fire; the $y$ terms represent attrition owing to area fire. Note that the algebraic forms chosen are consistent with current perceptions of aggregated large scale combat.

Now consider sources of noise, e.g., that at least arise from PD, PA, PH, PK, etc. Furthermore, such noise likely has its own functional dependencies, e.g., possibly being proportional to the numbers of units involved in the combat. Now we write

$$
\begin{aligned}
\frac{\Delta B T}{\Delta t} \equiv \dot{B} T= & x_{R T}^{B T} R T+y_{R T}^{B T} R T B T+x_{R B M P}^{B T} R B M P+y_{R B M P}^{B T} R B M P B T \\
& +z_{B T}^{B T} B T \eta_{B T}^{B T}+z_{R T}^{B T} \eta_{R T}^{B T}+z_{R B M P}^{B T} \eta_{R B M P}^{B T}
\end{aligned}
$$

where the $\eta$ represent sources of (white) noise (in the Itô prepoint discretization discussed below). The noise terms are taken to be log normal (multiplicative) noise for the diagonal terms and additive noise for the off-diagonal terms. The diagonal $z$ term $\left(z_{B T}^{B T}\right)$ represents uncertainty associated with the target $B T$, and the off-diagonal $z$ terms represent uncertainty associated with the shooters $R T$ and $R B M P$. The $x$ and $y$ are constrained such that each term is bounded by the mean of the KVS, averaged over all time and 
trajectories of similar scenarios; similarly, each $z$ term is constrained to be bounded by the variance of the KVS. The methodology presented here can accommodate any other nonlinear functional forms, and any other variables that can be reasonably represented by such rate equations, e.g., expenditures of ammunition or bytes of communication. ${ }^{12}$ Variables that cannot be so represented, e.g., terrain, $\mathrm{C}^{3}$, weather, etc., must be considered as "super-variables" that specify the overall context for the above set of rate equations.

Equations similar to the $\dot{B T}$ equation are also written for $\dot{R T}, R \dot{B M P}, B \dot{A P C}$, and $B T \dot{O} W$. Only $x$ and $y$ that reflect possible nonzero entries in the KVS are free to be used for the fitting procedure. For example, since Janus(T) does not permit direct-fire fratricide, such terms are set to zero. In most NTC scenarios, fratricide typically is negligible. Nondiagonal noise terms give rise to correlations in the covariance matrix. Thus, we have

$$
\begin{gathered}
M^{G}=\{R T, R B M P, B T, B A P C, B T O W\} \\
M^{G}=g^{G}+\sum_{i} \hat{g}_{i}^{G} \eta^{i} \\
\hat{g}_{i}= \begin{cases}z_{i}^{G} M^{G}, & i=G \\
z_{i}^{G}, & i \neq G\end{cases}
\end{gathered}
$$

\section{B. Problems in Lanchester Theory}

Quasilinear deterministic mathematical modeling is not only a popular theoretical occupation, but many wargames, e.g., joint Theater Level Simulation (JTLS), use such equations as the primary algorithm to drive the interactions between opposing forces.

In its simplest form, this kind of mathematical modeling is known as Lanchester theory:

$$
\begin{aligned}
& \dot{r}=\mathrm{d} r / \mathrm{d} t=x_{r} b+y_{r} r b \\
& \dot{b}=\mathrm{d} b / \mathrm{d} t=x_{b} r+y_{b} b r
\end{aligned}
$$

where $r$ and $b$ represent red and blue variables, and the $x$ and $y$ are parameters that somehow should be fit to actual data.

It is well known, or should be well known, that it is notoriously difficult, if not impossible, to use the simple Lanchester equations to mathematically model any real data with any reasonable degree of precision. These equations perhaps are useful to discuss some gross systematics, but it is discouraging to accept that, for example, a procurement decision involving billions of dollars of national resources could hinge on mathematical models dependent on Lanchester theory.

Some investigators have gone further, and amassed historical data to claim that there is absolutely no foundation for believing that Lanchester theory has anything to do with reality. ${ }^{13}$ However, although there is some truth to the above criticisms, the above conclusions do not sit comfortably with other vast stores of human experience. Indeed, this controversy is just one example that supports the necessity of having human intervention in the best of $\mathrm{C}^{2}$ plans, no matter how (seemingly) sophisticated analysis supports conclusions contrary to human judgment. ${ }^{10}$ That is, when dealing with a dynamic complex system, intuition and analysis must join together to forge acceptable solutions. The purpose of good theory and good data should be to ease the burden placed on the human decision maker, and to enable better decisions to be made.

We need better nonlinear stochastic theory than provided by Lanchester theory. ${ }^{14}$ Just as important, as we have numerically detailed in this project, data to test such models must be "time dense'," i.e., available on time scales of minutes, not days or weeks as is typically given by actual combat records. Also, the kind of data required, KVSs, is hard enough to extract from laser-recorded exercises, e.g., at NTC; getting such data from combat journals is unrealistic.

\section{Nonlinear Stochastic Processes}

Aggregation problems in such nonlinear nonequilibrium systems typically are "solved" (accommodated) by having new entities/languages developed at these disparate scales to efficiently pass information back and forth. $^{92,15,16}$ This is quite different from the nature of quasi-equilibrium quasi-linear systems, where thermodynamic or cybernetic approaches are possible; these approaches typically fail for nonequilibrium nonlinear systems.

In the late 1970's, mathematical physicists discovered that they could develop statistical mechanical theories from algebraic functional forms 


$$
\begin{aligned}
\dot{r} & =f_{r}(r, b)+\sum_{i} \hat{g}_{r}^{i}(r, b) \eta_{i} \\
\dot{b} & =f_{b}(b, r)+\sum_{i} \hat{g}_{b}^{i}(b, r) \eta_{i}
\end{aligned}
$$

where the $\hat{g}$ and $f$ are general nonlinear algebraic functions of the variables $r$ and $b .{ }^{17-22}$ The $f$ are referred to as the (deterministic) drifts, and the square of the $\hat{g}$ are related to the diffusions (fluctuations). In fact, the statistical mechanics can be developed for any number of variables, not just two. The $\eta$ are sources of Gaussian-Markovian noise, often referred to as "white noise." The inclusion of the $\hat{g}$, called "multiplicative" noise, has been shown to very well model mathematically and physically other forms of noise, e.g., shot noise, colored noise, dichotomic noise. ${ }^{23-25}$

The ability to include many variables also permits a "field theory" to be developed, e.g., to have sets of $(r, b)$ variables (and their rate equations) at many grid points, thereby permitting the exploration of spatial-temporal patterns in $r$ and $b$ variables. This gives the possibility of mathematically modeling the dynamic interactions across a large terrain. Modern computer capabilities are daily brought to bear on similar problems of this magnitude.

These new methods of nonlinear statistical mechanics have been applied to complex large scale physical problems, demonstrating that empirical data can be described by of these algebraic functional forms. Success was gained for large scale systems in neuroscience, in a series of papers on statistical mechanics of neocortical interactions (SMNI) ${ }^{26-40}$ in nuclear physics, ${ }^{41,42}$ and in financial markets. ${ }^{43-45}$ These have been proposed for problems in $\mathrm{C}^{3}$. $^{2}, 15,46-48$

Thus, now we can investigate various choices of $f$ and $\hat{g}$ to see if algebraic functional forms close to the Lanchester forms can actually fit the data. In physics, this is the standard phenomenological approach to discovering and encoding knowledge and empirical data, i.e., fitting algebraic functional forms that lend themselves to physical interpretation. This gives more confidence when extrapolating to new scenarios, exactly the issue in building confidence in combat computer models.

The utility of these algebraic functional forms goes further beyond their being able to fit sets of data. There is an equivalent representation to these stochastic differential equations, called a "path integral" representation for the long-time probability distribution of the variables. This short-time conditional probability distribution is driven by a "Lagrangian," that can be thought of as a dynamic algebraic "cost" function. The path integral representation for the long-time distribution possesses a variational principle, which means that simple graphs of the algebraic cost-function give a correct intuitive view of the most likely states of the variables, and of their statistical moments, e.g., heights being first moments (likely states) and widths being second moments (uncertainties). Like a ball bouncing about a terrain of hills and valleys, one can quickly visualize the nature of dynamically unfolding $r$ and $b$ states.

Especially because we are trying to mathematically model sparse and poor data, different drift and diffusion algebraic functions can give approximately the same algebraic cost-function when fitting shorttime probability distributions to data. The calculation of long-time distributions permits a better choice of the best algebraic functions, i.e., those which best follow the data through a predetermined epoch of battle. Thus, dynamic physical mechanisms, beyond simple "line" and "area" firing terms, can be identified. Afterwards, if there are closely competitive algebraic functions, they can be more precisely assessed by calculating higher algebraic correlation functions from the probability distribution.

It must be clearly stated that, like any other theory applied to a complex system, these methods have their limitations, and they are not a panacea for all systems. For example, probability theory itself is not a complete description when applied to categories of subjective "possibilities" of information. ${ }^{49,50}$ Other non-stochastic issues are likely appropriate for determining other types of causal relationships, e.g., the importance of reconnaissance to success of missions. ${ }^{51}$ These statistical mechanical methods appear to be appropriate for describing stochastic large scale combat systems. The details of our studies will help to determine the correctness of this premise.

As discussed above, the mathematical representation most familiar to other modelers is a system of stochastic rate equations, often referred to as Langevin equations. From the Langevin equations, other models may be derived, such as the times-series model and the Kalman filter method of control theory. However, in the process of this transformation, the Markovian description typically is lost by projection onto a smaller state space. ${ }^{52,53}$ This work only considers multiplicative Gaussian noise, including the limit of weak colored noise. ${ }^{24}$ These methods are not conveniently used for other sources of noise, e.g., Poisson processes or Bernoulli processes. It remains to be seen if multiplicative noise can emulate these processes 
in the empirical ranges of interest, in some reasonable limits. ${ }^{25}$ At this time, certainly the proper inclusion of multiplicative noise, using parameters fit to data to model general sources of noise, is preferable to improper inclusion or exclusion of any noise.

\section{Algebraic Complexity Yields Simple Intuitive Results}

Consider a multivariate system, but with the multivariate variance a general nonlinear function of the variables. The Einstein summation convention helps to compact the equations, whereby repeated indices in factors are to be summed over.

The Itô (prepoint) discretization for a system of stochastic differential equations is defined by

$$
\begin{gathered}
\bar{t}_{s} \in\left[t_{s}, t_{s}+\Delta t\right] \\
M\left(\bar{t}_{s}\right)=M\left(t_{s}\right) \\
\dot{M}\left(\bar{t}_{s}\right)=M\left(t_{s+1}\right)-M\left(t_{s}\right)
\end{gathered}
$$

The stochastic equations are then written as

$$
\begin{aligned}
\dot{M}^{G} & =f^{G}+\hat{g}_{i}^{G} \eta^{i} \\
i & =1, \cdots, \Xi \\
G & =1, \cdots, \Theta
\end{aligned}
$$

The operator ordering (of the $\partial / \partial M^{G}$ operators) in the Fokker-Planck equation corresponding to this discretization is

$$
\begin{gathered}
\frac{\partial P}{\partial t}=V P+\frac{\partial\left(-g^{G} P\right)}{\partial M^{G}}+\frac{1}{2} \frac{\partial^{2}\left(g^{G G^{\prime}} P\right)}{\partial M^{G} \partial M^{G^{\prime}}} \\
g^{G}=f^{G}+\frac{1}{2} \hat{g}_{i}^{G^{\prime}} \frac{\partial \hat{g}_{i}^{G}}{\partial M^{G^{\prime}}} \\
g^{G G^{\prime}}=\hat{g}_{i}^{G} \hat{g}_{i}^{G}
\end{gathered}
$$

The Lagrangian corresponding to this Fokker-Planck and set of Langevin equations may be written in the Stratonovich (midpoint) representation, corresponding to

$$
M\left(\bar{t}_{s}\right)=\frac{1}{2}\left[M\left(t_{s+1}\right)+M\left(t_{s}\right)\right]
$$

This discretization can be used to define a Feynman Lagrangian $L$ that possesses a variational principle, and which explicitly portrays the underlying Riemannian geometry induced by the metric tensor $g_{G G^{\prime}}$, calculated to be the inverse of the covariance matrix.

$$
\begin{aligned}
& P=\int \cdots \int \underline{D} M \exp \left(-\sum_{s=0}^{u} \Delta t L_{s}\right) \\
& \underline{D} M=g_{0_{+}}^{1 / 2}(2 \pi \Delta t)^{-1 / 2} \prod_{s=1}^{u} g_{s_{+}}^{1 / 2} \prod_{G=1}^{\Theta}(2 \pi \Delta t)^{-1 / 2} \mathrm{~d} M_{s}^{G} \\
& \int \mathrm{d} M_{s}^{G} \rightarrow \sum_{\alpha=1}^{N^{G}} \Delta M_{\alpha s}^{G}, M_{0}^{G}=M_{t_{0}}^{G}, M_{u+1}^{G}=M_{t}^{G} \\
& L=\frac{1}{2}\left(\dot{M}^{G}-h^{G}\right) g_{G G^{\prime}}\left(\dot{M}^{G^{\prime}}-h^{G^{\prime}}\right)+\frac{1}{2} h_{; G}^{G}+R / 6-V \\
& {[\cdots]_{, G}=\frac{\partial[\cdots]}{\partial M^{G}}} \\
& h^{G}=g^{G}-\frac{1}{2} g^{-1 / 2}\left(g^{1 / 2} g^{G G^{\prime}}\right)_{G^{\prime}} \\
& g_{G G^{\prime}}=\left(g^{G G^{\prime}}\right)^{-1} \\
& g_{s}\left[M^{G}\left(\bar{t}_{s}\right), \bar{t}_{s}\right]=\operatorname{det}\left(g_{G G^{\prime}}\right)_{s}, g_{s_{+}}=g_{S}\left[M_{s+1}^{G}, \bar{t}_{s}\right] \\
& h_{; G}^{G}=h_{G}^{G}+\Gamma_{G F}^{F} h^{G}=g^{-1 / 2}\left(g^{1 / 2} h^{G}\right)_{, G} \\
& \Gamma_{J K}^{F} \equiv g^{L F}[J K, L]=g^{L F}\left(g_{J L, K}+g_{K L, J}-g_{J K, L}\right) \\
& R=g^{J L} R_{J L}=g^{J L} g^{J K} R_{F J K L} \\
& R_{F J K L}=\frac{1}{2}\left(g_{F K, J L}-g_{J K, F L}-g_{F L, J K}+g_{J L, F K}\right)+g_{M N}\left(\Gamma_{F K}^{M} \Gamma_{J L}^{N}-\Gamma_{F L}^{M} \Gamma_{J K}^{N}\right)
\end{aligned}
$$

A "potential" term $V$ is included, e.g., which might arise to simulate boundary conditions.

Because of the presence of multiplicative noise, the Langevin system differs in its Itô (prepoint) and Stratonovich (midpoint) discretizations. The midpoint-discretized covariant description, in terms of the Feynman Lagrangian, is defined such that (arbitrary) fluctuations occur about solutions to the Euler- 
Lagrange variational equations. In contrast, the usual Itô and corresponding Stratonovich discretizations are defined such that the path integral reduces to the Fokker-Planck equation in the weak-noise limit. The term $R / 6$ in the Feynman Lagrangian includes a contribution of $R / 12$ from the WKBJ approximation ${ }^{54}$ (named after Wentzel, Kramers, Brillouin, and Jefferys) to the same order of $(\Delta t)^{3 / 2} .^{21}$

Now, consider the generalization to many cells. In the absence of any further information about the system, this increases the number of variables, from the set $\{G\}$ to the set $\{G, v\}$.

A different prepoint discretization for the same probability distribution $P$, gives a much simpler algebraic form, but the Lagrangian $L^{\prime}$ so specified does not satisfy a variational principle useful for moderate to large noise.

$$
L^{\prime}=\frac{1}{2}\left(\dot{M}^{G}-g^{G}\right) g_{G G^{\prime}}\left(\dot{M}^{G^{\prime}}-g^{G^{\prime}}\right)
$$

Still, this prepoint-discretized form has been quite useful in all systems examined thus far, simply requiring a somewhat finer numerical mesh.

It must be emphasized that the output need not be confined to complex algebraic forms or tables of numbers. Because $L$ possesses a variational principle, sets of contour graphs, at different long-time epochs of the path integral of $P$ over its $r$ and $b$ variables at all intermediate times, give a visually intuitive and accurate decision-aid to view the dynamic evolution of the scenario.

This Lagrangian approach to combat dynamics permits a quantitative assessment of concepts previously only loosely defined.

Momentum:

Mass:

$$
\Pi^{G}=\frac{\partial L}{\partial\left(\partial M^{G} / \partial t\right)}
$$

Force:

$$
g_{G G^{\prime}}=\frac{\partial L}{\partial\left(\partial M^{G} / \partial t\right) \partial\left(\partial M^{G^{\prime}} / \partial t\right)}
$$

$$
F=m a: \quad \frac{\partial L}{\partial M^{G}}
$$

where $M^{G}$ are the variables and $L$ is the Lagrangian. These relationships are derived and are valid at each spatial-temporal point of $M^{G}$. Reduction to other mathematical and physics modeling can be achieved after fitting realistic exercise and/or simulation data.

These physical entities provide another form of intuitive, but quantitatively precise, presentation of these analyses. A visual example is given below.

\section{Numerical Implementation}

\section{A. Fitting Parameters}

The five coupled stochastic differential equations, for variables $M^{G}=\{R T, R B M P, B T, B A P C, B T O W\}$, can be represented equivalently by a short-time conditional probability distribution $P$ in terms of a Lagrangian $L$ :

$$
P(R \cdot, B \cdot ; t+\Delta t \mid R \cdot, B \cdot ; t)=\frac{1}{(2 \pi \Delta t)^{5 / 2} \sigma^{1 / 2}} \exp (-L \Delta t)
$$

where $\sigma$ is the determinant of the inverse of the covariance matrix, the metric matrix of this space, " $R$." represents $\{R T, R B M P\}$, and " $B$." represents $\{B T, B A P C, B T O W\}$. (Here, the prepoint discretization is used, which hides the Riemannian corrections explicit in the midpoint discretized Feynman Lagrangian; only the latter representation possesses a variational principle useful for arbitrary noise.)

This defines a scalar "dynamic cost function," $C(x, y, z)$,

$$
C(x, y, z)=L \Delta t+\frac{5}{2} \ln (2 \pi \Delta t)+\frac{1}{2} \ln \sigma
$$

which can be used with the very fast simulated reannealing (VFSR) algorithm ${ }^{55}$ further discussed below, to find the (statistically) best fit of $\{x, y, z\}$ to the data. 
The form for the Lagrangian $L$ and the determinant of the metric $\sigma$ to be used for the cost function $C$ is

$$
\begin{gathered}
L=\sum_{G} \sum_{G^{\prime}} \frac{\left(\dot{M}^{G}-g^{G}\right)\left(\dot{M}^{G^{\prime}}-g^{G^{\prime}}\right)}{2 g^{G G^{\prime}}} \\
\sigma=\operatorname{det}\left(g_{G G^{\prime}}\right) \\
\left(g_{G G^{\prime}}\right)=\left(g^{G G^{\prime}}\right)^{-1} \\
g^{G G^{\prime}}=\sum_{i} \hat{g}_{i}^{G} \hat{g}_{i}^{G^{\prime}}
\end{gathered}
$$

Generated choices for $\{x, y, z\}$ are constrained by empirical KVSs (taken from exercises or from computer simulations of these exercises)

$$
\begin{gathered}
g^{G}(t) \leq n^{G}<\Delta M^{G}(t)> \\
\hat{g}_{i}^{G}(t) \leq n_{i}^{G}\left[<\left(\Delta M^{G}(t)\right)^{2}>\right]^{1 / 2}
\end{gathered}
$$

where $n^{G}$ and $n_{i}^{G}$ are the number of terms in $g^{G}$ and $\hat{g}_{i}^{G}$, respectively, and averages $<\cdot>$ are taken over all time epochs and trajectories of similar scenarios.

If there are competing mathematical forms, then it is advantageous to use the path integral to calculate the long-time evolution of $P{ }^{12}$ Experience has demonstrated that, since $P$ is exponentially sensitive to changes in $L$, the long-time correlations derived from theory, measured against the empirical data, is a viable and expedient way of rejecting models not in accord with empirical evidence.

Note that the use of the path integral is a posteriori to the short-time fitting process, and is a subsidiary physical constraint on the mathematical models to judge their internal soundness and suitability for attempts to extrapolate to other scenarios.

\section{B. Combat Power Scores}

After the $\{x, y, z$,$\} are fit to the data and a mathematical model is selected, another fit can be super-$ imposed to find the effective "combat scores," defined here as the relative contribution of each system to the specific class of scenarios in question. Using a fundamental property of probability distributions, a probability distribution $P_{A}(q)$ of aggregated variables $q_{1}+q_{2}$ can be obtained from the probability distribution for $P\left(q_{1}, q_{2}\right)$

$$
P_{A}\left(q=q_{1}+q_{2}\right)=\int \mathrm{d} q_{1} \mathrm{~d} q_{2} P\left(q_{1}, q_{2}\right) \delta\left(q-q_{1}-q_{2}\right)
$$

where $\delta(\cdot)$ is the Dirac delta function.

Thus, we calculate the aggregated conditional probability

$$
\begin{gathered}
P_{A}(r, b ; t+\Delta t \mid R \cdot, B \cdot ;) \\
=\int \mathrm{d} R T \mathrm{~d} R B M P \mathrm{~d} B T \mathrm{~d} B A P C \mathrm{~d} B T O W P(R \cdot, B \cdot ;+\Delta t \mid R \cdot, B \cdot ; t) \\
\times \delta\left(r-w_{R T}^{r} R T-w_{R B M P}^{r} R B M P\right) \\
\times \delta\left(b-w_{R T}^{b} B T-w_{B A P C}^{b} B A P C-w_{B T O W}^{b} B T O W\right)
\end{gathered}
$$

where the $w$ represent the desired combat scores. After the $\{x, y, z\}$ have been fitted, the new parameters $\{w\}$ can be fit to the data by maximizing the cost function $C^{\prime}(w)$ using VFSR,

$$
C^{\prime}(w)=-\ln P_{A}
$$

Note that the simple linear aggregation by systems above can be generalized to nonlinear functions, thereby taking into account synergistic interactions among systems that contribute to overall combat effectiveness.

We will be able to explore the possibility of developing human factors combat power scores, since we will be similarly including human-factors variables in such equations, as discussed below.

\section{Very Fast Simulated Reannealing}

Two major computer codes have been developed, which are key tools for use of this approach to mathematically model combat data. The first code, very fast simulated reannealing (VFSR), ${ }^{55}$ fits shorttime probability distributions to empirical data, using a most-likelihood technique on the Lagrangian. This algorithm has been developed to fit empirical data to a theoretical cost function over a $D$ dimensional parameter space, ${ }^{55}$ adapting for varying sensitivities of parameters during the fit. The annealing schedule for the "temperatures" (artificial fluctuation parameters) $T_{i}$ decrease exponentially in "time" (cycle-number of iterative process) $k$, i.e., $T_{i}=T_{i 0} \exp \left(-c_{i} k^{1 / D}\right)$. 
Heuristic arguments have been developed to demonstrate that this algorithm is faster than the fast Cauchy annealing: ${ }^{56} T_{i}=T_{0} / k$, and much faster than Boltzmann annealing: ${ }^{57} T_{i}=T_{0} / \ln k$. To be more specific, the $k$ th estimate of parameter $\alpha^{i}$,

$$
\alpha_{k}^{i} \in\left[A_{i}, B_{i}\right]
$$

is used with the random variable $x^{i}$ to get the $k+1$ th estimate,

$$
\begin{aligned}
\alpha_{k+1}^{i}= & \alpha_{k}^{i}+x^{i}\left(B_{i}-A_{i}\right) \\
& x^{i} \in[-1,1]
\end{aligned}
$$

Define the generating function

$$
g_{T}(x)=\prod_{i=1}^{D} \frac{1}{2 \ln \left(1+1 / T_{i}\right)\left(\left|x^{i}\right|+T_{i}\right)} \equiv \prod_{i=1}^{D} g_{T}^{i}\left(x^{i}\right)
$$

The cost-functions $L$ we are exploring are of the form

$$
\begin{gathered}
h(M ; \alpha)=\exp (-\underline{L} / T) \\
\underline{L}=L \Delta t+\frac{1}{2} \ln \left(2 \pi \Delta t g_{t}^{2}\right)
\end{gathered}
$$

where $L$ is a Lagrangian with dynamic variables $M(t)$, and parameter coefficients $\alpha$ to be fit to data. The $g_{t}$ is the determinant of the metric. Note that the use of $L$ is not equivalent to doing a simple least squares fit on $M(t+\Delta t)$.

Recently, a comparison of VFSR was made with another popular approach, Genetic Algorithms (GA). ${ }^{58}$ GA previously has been demonstrated to be competitive with other standard Boltzmann-type simulated annealing techniques. Presenting a suite of six standard test functions to GA and VFSR codes from previous studies, without any additional fine tuning, strongly suggests that VFSR can be expected to be orders of magnitude more efficient than GA. Other studies have shown VSFR to be superior to other simulated annealing techniques. ${ }^{59,60}$ A new algorithm has been outlined, combining the strengths with other powerful algorithms. ${ }^{39}$ Since VFSR was recoded and made publicly available, many groups worldwide have made it the algorithm of choice for complex systems. ${ }^{61}$

\section{Path Integral}

The second code develops the long-time probability distribution from the Lagrangian fit by the first code. A robust and accurate histogram-based (nonMonte Carlo) path integral algorithm to calculate the long-time probability distribution has been developed to handle nonlinear Lagrangians, ${ }^{15,62-64}$ including a two variable code for additive and multiplicative cases.

The histogram procedure recognizes that the distribution can be numerically approximated to a high degree of accuracy as the sum of rectangles at points $M_{i}$ of height $P_{i}$ and width $\Delta M_{i}$. For convenience, just consider a one dimensional system. The above path integral representation can be rewritten, for each of its intermediate integrals, as

$$
\begin{aligned}
P(M ; t+\Delta t) & =\int \mathrm{d} M^{\prime}\left[g_{s}^{1 / 2}(2 \pi \Delta t)^{-1 / 2} \exp \left(-L_{s} \Delta t\right)\right] P\left(M^{\prime} ; t\right) \\
& \equiv \int \mathrm{d} M^{\prime} G\left(M, M^{\prime} ; \Delta t\right) P\left(M^{\prime} ; t\right) \\
& P(M ; t)=\sum_{i=1}^{N} \pi\left(M-M_{i}\right) P_{i}(t) \\
\pi\left(M-M_{i}\right)= & \left\{\begin{array}{l}
1,\left(M_{i}-\frac{1}{2} \Delta M_{i-1}\right) \leq M \leq\left(M_{i}+\frac{1}{2} \Delta M_{i}\right) \\
0, \text { otherwise }
\end{array}\right.
\end{aligned}
$$

which yields

$$
T_{i j}(\Delta t)=\frac{2 \quad P_{i}(t+\Delta t)=T_{i j}(\Delta t) P_{j}(t)}{\Delta M_{i-1}+\Delta M_{i}} \int_{M_{i}-\Delta M_{i-1} / 2}^{M_{i}+\Delta M_{i} / 2} \mathrm{~d} M \int_{M_{j}-\Delta M_{j-1} / 2}^{M_{j}+\Delta M_{j} / 2} \mathrm{~d} M^{\prime} G\left(M, M^{\prime} ; \Delta t\right)
$$

$T_{i j}$ is a banded matrix representing the Gaussian nature of the short-time probability centered about the (varying) drift. 
This histogram procedure has been extended to two dimensions for this combat analysis, that is, using a matrix $T_{i j k l}$, ${ }^{15}$ e.g., similar to the use of the $A$ matrix in the previous section. Explicit dependence of $L$ on time $t$ also can be included without complications. We see no problems in extending it to other dimensions, other than care must be used in developing the mesh in $\Delta M$, which is dependent on the diffusion matrix.

Fitting data with the short-time probability distribution, effectively using an integral over this epoch, permits the use of coarser meshes than the corresponding stochastic differential equation. The coarser resolution is appropriate, typically required, for numerical solution of the time dependent path integral: By considering the contributions to the first and second moments of $\Delta M^{G}$ for small time slices $\theta$, conditions on the time and variable meshes can be derived. ${ }^{62}$ The time slice is determined by $\theta \leq \bar{L}^{-1}$, where $\bar{L}$ is the "static" Lagrangian with $\mathrm{d} M^{G} / \mathrm{d} t=0$, throughout the ranges of $M^{G}$ giving the most important contributions to the probability distribution $P$. The variable mesh, a function of $M^{G}$, is optimally chosen such that $\Delta M^{G}$ is measured by the covariance $g^{G G^{\prime}}$, or $\Delta M^{G} \sim\left(g^{G G} \theta\right)^{1 / 2}$.

As is true for many systems described by such stochastic equations, the equations themselves are but a part of the mathematical model, being complemented by boundary conditions. These are usually difficult to implement in differential equations. That is, codes that the author has seen for Lanchester-type systems do not include such conditions as bounds on numbers of units available. However, in the path integral formalism typically these boundary conditions can be readily and properly included, ${ }^{64}$ and we have done so.

\section{E. Modeling of National Training Center}

The "kills" attrition data from NTC and our Janus(T)/NTC simulation at once looks strikingly similar during the force-on-force part of the combat (Fig. 1). Note that we are fitting (only half) the middle part of the engagement, where the slope of attrition is very steep (and almost linear on the given scale), i.e., the "force on force" part of the engagement. The second half of the data must be predicted by our models.

From the single NTC trajectory qualified to date, seven 5-min intervals in the middle of the battle were selected. From six Janus(T) runs, similar force-on-force time epochs were identified, for a total of 42 data points. In the following fits, $r$ represents red tanks, and $b$ represents blue tanks.

Fitting NTC data to an additive noise model, a cost function of 2.08 gave

$$
\begin{aligned}
& \dot{r}=-2.49 \times 10^{-5} b-4.97 \times 10^{-4} b r+0.320 \eta_{r} \\
& \dot{b}=-2.28 \times 10^{-3} r-3.23 \times 10^{-4} r b+0.303 \eta_{b}
\end{aligned}
$$

Fitting NTC data to a multiplicative noise model, a cost function of 2.16 gave

$$
\begin{aligned}
& \dot{r}=-5.69 \times 10^{-5} b-4.70 \times 10^{-4} b r+1.06 \times 10^{-2}(1+r) \eta_{r} \\
& \dot{b}=-5.70 \times 10^{-4} r-4.17 \times 10^{-4} r b+1.73 \times 10^{-2}(1+b) \eta_{b}
\end{aligned}
$$

Fitting Janus(T) data to an additive noise model, a cost function of 3.53 gave

$$
\begin{aligned}
& \dot{r}=-2.15 \times 10^{-5} b-5.13 \times 10^{-4} b r+0.530 \eta_{r} \\
& \dot{b}=-5.65 \times 10^{-3} r-3.98 \times 10^{-4} r b+0.784 \eta_{b}
\end{aligned}
$$

Fitting Janus(T) data to a multiplicative noise model, a cost function of 3.42 gave

$$
\begin{aligned}
& \dot{r}=-2.81 \times 10^{-4} b-5.04 \times 10^{-4} b r+1.58 \times 10^{-2}(1+r) \eta_{r} \\
& \dot{b}=-3.90 \times 10^{-3} r-5.04 \times 10^{-4} r b+3.58 \times 10^{-2}(1+b) \eta_{b}
\end{aligned}
$$

This comparison illustrates that two different models about equally fit the short-time distribution. The multiplicative noise model shows that approximately a factor of 100 of the noise might be "divided out," or understood in terms of the physical log normal mechanism.

To discern which model best fits the data, we turn to the path integral calculation of the long-time distribution, to see which model best follows the actual data. Figure 2 presents the long-time probability of finding values of these forces. In general, the probability will be a highly nonlinear algebraic function, and there will be multiple peaks and valleys.

Figures 3 and 4 give the means and variances of tank attrition from the Janus(T) and NTC databases. Since we now have only one NTC mission qualified, the variance of deviation from the mean is not really significant; it is given only to illustrate our approach that will be applied to more NTC missions as they are qualified and aggregated. Note that only the blue Janus(T) variances of the additive noise model are consistent with the NTC data. 


\section{F. Discussion of Study}

Data from 35 to 70 minutes was used for the short-time fit. The path integral code was used to calculate the long-time evolution of this fitted short-time (5-min) distribution from 35 to beyond 70 min. This serves to compare long-time correlations in the mathematical model vs the data, and to help judge extrapolation past the data used for the short-time fits. More data and work are required to find a better (or best?) algebraic form. The resulting form is required for input into higher echelon models. As more NTC data becomes available (other NTC missions are in the process of being qualified, wargamed, and analyzed), we will be able to judge the best models with respect to how well they extrapolate across slightly different combat missions.

\section{Chaos or Noise?}

Given the context of current studies in complex nonlinear systems, the question can be asked: What if combat has chaotic mechanisms that overshadow the above stochastic considerations? The real issue is whether the scatter in data can be distinguished from that due to noise or chaos. Several studies have been proposed with regard to comparing chaos to simple filtered (colored) noise. ${ }^{65-67}$

The combat analysis was possible only because now we had data on combat exercises from the NTC of sufficient temporal density to attempt dynamical mathematical modeling. The criteria used to (not) determine chaos in this dynamical system is the nature of propagation of uncertainty, i.e., the variance. For example, following by now standard arguments, propagation of uncertainty may be considered as (1) diminishing, (2) increasing additively, (3) or increasing multiplicatively. An example of (1) is the evolution of a system to an attractor, e.g., a book dropped onto the floor from various heights reaches the same point no matter what the spread in initial conditions. An example of (2) is the propagation of error in a clock, a cyclic system. Examples of (3) are chaotic systems, of which very few real systems have been shown to belong. An example of (3) is the scattering of a particle in a box whose center contains a sphere boundary: When a spread of initial conditions is considered for the particle to scatter from the sphere, when its trajectories are aligned to strike the sphere at a distance from its center greater that the diameter, the spread in scattering is a factor of about three greater than the initial spread.

In our analysis of NTC data, we were able to fit the short-time attrition epochs (determined to be about 5 min from mesh considerations determined by the nature of the Lagrangian) with short-time nonlinear Gaussian-Markovian probability distributions with a resolution comparable to the spread in data. When we computed the long-time path integral from some point (spread) at the beginning of the battle, we found that we could readily find a form of the Lagrangian that made physical sense and that also fit the multivariate variances as well as the means at each point in time of the rest of the combat interval; i.e., there was not any degree of sensitivity to initial conditions that prevented us from "predicting" the longtime means and variances of the system. Of course, since the system is dissipative, there is a strong tendency for all moments to diminish in time, but in fact this combat is of sufficiently modest duration (typically $1-2 \mathrm{~h}$ ) that variances do increase somewhat during the middle of the battle.

In summary, this battalion-regiment scale of battle does not seem to possess chaos. Similar to serious work undertaken in several fields, ${ }^{68,69}$ here too, the impulse to cry "chaos!" in combat appears to have been shown to be premature. It is not supported by the facts, tentative as they are because of sparse data. Of course, some other combat conditions might show some elements of chaos in some spatial-temporal domain, and then the resolution of the analysis would determine the influence of that chaos on that combat scenario.

A more purposeful project is to compare stochastic with deterministic models of combat data. Today much attention is turning to the use of deterministic chaotic models for short-time predictions of systems. For example, if only short-time predictions are required, and if a deterministic chaotic model could well describe stochastic data within these epochs, then this model might be more computationally efficient instead of a more "correct" stochastic model that would be necessary for long-time predictions. The scales of time involved are of course system dependent, and the deterministic chaotic modeling of noisy data is still in its infancy. ${ }^{70}$

\section{Statistical Mechanics of Combat with Human Factors}




\section{A. Rationale}

In many complex activities involving interactions between human and inanimate systems, e.g., modern combat, the nonlinear synergies capable between these systems make it impossible to separate their influences from the total scenario. However, their relationships and functional dependencies still might be amenable to explicit scientific description. Working with Sworder at University of California at San Diego, we are developing the approach below. ${ }^{47}$

For example, if $h(t)$ could be determined to be a (time dependent) human factor, and if $x(t)$ could be determined to be an inanimate factor, then one could imagine that a "cost function" $C$ fitting data from a specific class of combat scenarios could be fit by a probability distribution $P[C]$ (emphasizing the uncertainty and "noise" in both systems). For specificity, consider the completely arbitrary distribution selected only for purposes of illustration.

$$
\begin{gathered}
P[C]=N \exp (-C) \\
C(x, h ; t)=\frac{\left(\mathrm{d} x / \mathrm{d} t+3.04 x h^{2}+0.21 x^{2}\right)^{2}}{\left(2.84+0.133 h^{2} x^{2}\right)}+\frac{\left(\mathrm{d} h / \mathrm{d} t+5.10 x^{2}+1.13 h x^{2}\right)^{2}}{\left(4.21+0.071 h^{2} x^{2}\right)}
\end{gathered}
$$

where $N(x, h ; t)$ is a normalization factor for this distribution.

As discussed above, ${ }^{15}$ we have derived similar stochastic nonlinear forms, in terms of nonlinear stochastic time dependent inanimate combat variables (tank attrition, etc.), that fit quite well to data from exercises at NTC. We propose here to more explicitly include the human-factor variables relevant to decision making processes at NTC. The determination of such a cost function permits the accurate derivation of graphical aids to visualize the sensitivity of combat macrovariables (force, mass, momentum) as a function of the human decision making process. ${ }^{46}$

It is perhaps just as important for us to clearly state what we are not proposing. We are not proposing that the human-factors variables we will derive, e.g., in the sense of $h$, will be explicit representations of cognitive activity, such as attentional processes. Rather, these variables are to be considered metavariables representing the behavioral characteristics of human decision making, ${ }^{71}$ in the context of specific NTC scenarios. The above sensitivity measures of the decision making process perhaps comes closest with this methodology to explicit identification of human factors.

We believe that we can deliver normative (probabilistic) standards of a class(es) of NTC scenarios, by which specific unit decision making performance can be gauged within this context. Furthermore, our explicit representations of these human factors permits these equations to be used directly in many combat computer models, thereby increasing the utility of these computer models for training and analysis. The lack of human-factors algorithms in combat computer models is notorious. ${ }^{72-74}$

Our major thrust will be to identify and to interpret reasonable functional forms much more extensive and detailed than above. This project is new research territory and it will require extensive and intensive interaction between accumulating critical analyses and accumulating experience with operations at NTC.

The inclusion of human factors in a single equation is too naive to capture the essence of human decision making, even if we generalize $h$ and $x$ to include many variables from each opponent, e.g., $\tilde{h}$ and $\tilde{x}$ vectors. To include sharp bifurcations, e.g., alternative branching scenarios because of the perceptions of commanders and actions thereby taken at critical times in combat, we plan to fit a "tree" of distributions, each branch representing an alternative scenario.

Our rationale for this attempt, to generalize our previous NTC fits of inanimate variables, ${ }^{46}$ is based on our other work modeling the human decision maker controlling teleoperated robotic vehicles, a decision making process that is conceptually similar to the role of the commander in combat. ${ }^{71,75}$ We should thereby gain greater fidelity in our fits to NTC data by more explicitly including human factors.

A major thrust of our research will be to expand the linearized theory beyond that currently formulated, ${ }^{71}$ to include more robust nonlinear features of the underlying theory of human decision making. This approach is now possible because of the spin-off work in the previous project, ${ }^{46}$ i.e., developing the VFSR methodology to fit such nonlinear multivariate stochastic models. ${ }^{55}$

\section{B. Significant Aspects to be Studied}

We believe we are addressing the following issues: 
1) Human factors, especially in combat, are nonlinear. Nonlinearity arises for many reasons, ranging from synergies of human factors with physical systems, to multivalued decision trees depicting future states. The inclusion of realistic movements on realistic terrain typically presents a nonlinear spatial-temporal surface on which the variables evolve.

2) Human factors are stochastic. There are relatively separable influences on decision making, e.g., probabilities associated with detections, acquisitions, hits, and kills. Furthermore, especially in a given complex situation, not only will different people often make different decisions at different times, but, given the same opportunities, the same person often will make different decisions at different times. Therefore, we need multiple runs of similar situations in order to deduce these distributions. Such sets of data, albeit not ideal, are present at NTC.

3) Human factors are typically observed as "metavariables" of human performance. Especially because information possessed by decision makers is often incomplete or known to be at least partially incorrect, decision makers must make their decisions based on their current perceptions, their extrapolations to future perceptions, their perceptions of their opponent's perceptions, etc. These possibilities give rise to alternate behavioral states, in part contributing the nonlinearities and stochasticities discussed above.

4) Human factors are very context and domain dependent. Other approaches to human factors, e.g., in the field of artificial intelligence, are also converging on this realization. Analogical reasoning is often more efficient than logical deduction.

5) Even combat models with reasonable combat algorithms do not have reasonable human factors algorithms. Especially because of real time constraints, these computer models require relatively simple functional relationships if they are to include human factors at all. Our robust stochastic nonlinear approach permits us to identify (multiple) ranges of likely probable states, that then can be approximated by quasi-linear algebraic forms in each range. This forms the basis of an "expert" system that derives knowledge from objective fits of theoretical models to empirical data.

It is relevant to this paper to note that the projects discussed here, modeling NTC and Janus(T) as described above, and modeling teleoperated vehicles, both have brought powerful mathematical machinery to bear to the stages of numerical specificity with state-of-the-art successful description of realistic empirical data.

\section{Extensions to Other Systems}

\section{A. Amphibious Model}

One of the author's students wrote a nontechnical thesis on the mathematical methodology. ${ }^{76}$ Upton is looking at amphibious models, filling the gap in the spatial scales now using Air Force, Army, and Navy systems.

\section{B. Joint Janus Model}

Gallagher, another of the author's students, wrote a thesis documenting a Mideast Army-Navy joint scenario using a Battleship Battle Group with Tomahawk missiles supporting air-land combat. ${ }^{77}$ Balaco- $^{-}$ nis, another of the author's students, wrote a thesis documenting the extension of this joint concept to a NATO scenario, including studies of competitive strategies and integrated strike warfare, using two Carrier Battle Groups with Tomahawk and SLAM missiles, F-14 and A-6 tactical air support, and remotely piloted vehicles (Fig. 5). ${ }^{78}$

These projects have established a direction for further study (Fig. 6). We especially are aware of the necessity to include more Air Force systems.

\section{Issues of Higher Echelon Extrapolation}

After fitting data from microscopic unit interactions to mesoscopic equations at battalion-regiment level, these equations can be used to drive higher level macroscopic scenarios at corps and theater levels (Fig. 7). This mathematical aggregation is required for interpretation at multiple scales.

However, there are many issues yet to be resolved in using this approach. This requires approximately company-fidelity combat data from the unit interactions, e.g., barely the level obtained from NTC. It may be possible soon to obtain similar fidelity at division level, as NTC gears up for this scale of play. 
Currently there are four main approaches to modeling theater level combat. 1) Distribution of combat scenarios: The approach in this paper uses stochastic trajectories of high-fidelity interactions and develops stochastic distributions of lower-echelon scenarios. Linear MOFs are derived and battle nodes are coordinated for theater combat. 2) Distribution of system-system interactions: This approach, e.g., in COSAGE used at the Army Concepts Analysis Agency (CAA), uses statistical distributions of representative variables (including terrain and LOS) and distributions of system KVSs to develop attrition model for theater KVSs. 3) Deterministic combat scenarios: This approach, e.g., used in VIC at Training and Doctrine Command (TRADOC) Analysis Command (TRAC), White Sands Missile Range, New Mexico (TRAC-WSMR), develops KVSs from lower echelon scenarios and uses system KVSs for theater models. 4) Theater stochastic high fidelity model: This approach requires no aggregation, and studies all spatialtemporal scales simultaneously. This approach has regularly failed because of the huge computer resources required. Furthermore, aggregation is really required anyway, to simulate MOFs, MOEs, etc. required for cognitive decisions at various levels of command.

The important issues are: 1) sensitivity of theater models to different approaches, 2) inclusion/absorption of human factors into variables/parameters, 3) fidelity of representation of modern systems, e.g., cruise missiles (possessing short reaction times, large spatial coverage, and $\mathrm{C}^{3} \mathrm{I}$ at multiple scales), 4) statistical comparison of approaches, and 5) baselining of these approaches to some reality.

Response-surface methodologies and central composite design have been quite useful in a number of disciplines, effectively fitting (usually quadratic) algebraic forms to judicially selected instances of the appropriately scaled variables. Of course, the phenomenology underlying this approach might not work well for combat systems, but it should be tried; i.e., the nonlinear nature of combat might extend to supervariable space, requiring more sophisticated analysis than the typical regression on quadratic surfaces afforded by current implementations of this approach. Here, the methods of VFSR ${ }^{55,58}$ can be quite useful. At the least, this approach should be viewed as: given a fixed constraint of limited funds and time for a study, then there is a tradeoff between the depth (fidelity, etc.) of each scenario run vs breadth (number of runs, etc.).

We have suggested implementation these ideas, developing a mesoscopic filter of a joint model of models (JMOM). JMOM would take microscopic high fidelity combat information up from individual simulations/wargames into macroscopic quick response joint theater level simulations/wargames. This capability could greatly enhance the fidelity and validity of many high level analyses, e.g., of net assessments.

\section{Applications to Process Aggregated Information}

This statistical mechanics approach represents the mesoscale as a pattern processing computer. The underlying mathematical theory, i.e., the path integral approach, specifies a parallel-processing algorithm that statistically finds those parameter regions that contribute most to the overall probability distribution. This theoretical approach would not be very useful if we could not perform the calculations afforded by VFSR.

This theory represents a kind of "intuitive" algorithm, globally searching a large multivariate database to find parameter regions deserving more detailed local information processing. The derived probability distribution can be thought of as a filter, or processor, of incoming patterns of information; and this filter can be adapted, or updated, as it interacts with previously stored patterns of information.

As an example of how we can develop "intuitive" measures of performance, Figure 8 shows the derived force acting within the NTC system. These figures compare the NTC additive and multiplicative noise cases above, where we have graphed the force of red minus the force on blue. The difference between the red force and the blue force is portrayed as a function of number of vehicles. The solution of the evolution of the probability distribution gives the most likely number of vehicles existing at any time and their rates of attrition. Such figures at least serve to demonstrate that different models of combat can present quite different visual decision aids representing the nature of a particular scenario. This dramatizes the necessity for developing more sensitive algorithms to determine the proper models driving simulations and combat decision aids.

Two of the author's students, Connell, ${ }^{79}$ and Yost, ${ }^{80}$ have written theses examining multiple scales of interaction in large scale systems, including combat systems. These mathematical methods are quite general, and they have been applied to neuroscience, referenced previously as the SMNI papers, detailing 
properties of short-term memory derived from neuronal synaptic interactions, and calculating most likely frequencies observed in electroencephalographic (EEG) data and velocities of propagation of information across neocortex. We have detailed applications of this methodology to understand multiple scales of contributions to EEG data, developing software to perform accurate correlations between human behavioral states and EEG data. 37,38

Many systems require the processing of large sets of data, so large that it is generally conceded that it seems unlikely that even projected computer architectures will be adequate to handle these demands. ${ }^{81}$ Among these approaches offering some glimmer of hope are those that attempt to model the human information processing system, neocortex. "Neural nets" do not seem adequate. ${ }^{82}$ However, "neural nets" also have not demonstrated that they well model neocortex. Therefore, more investigations into the nature of neocortex is certainly still a plausible approach.

Recently, the SMNI methodology has been used to define an algorithm to construct a mesoscopic neural net (MNN), based on realistic neocortical processes and parameters, to record patterns of brain activity and to compute the evolution of this system. ${ }^{39}$ Furthermore, this new algorithm is quite generic, and can be used to similarly process information in other systems amenable to modeling by mathematical physics techniques alternatively described by path integral Lagrangians, Fokker-Planck equations, or Langevin rate equations, e.g., such as the combat analyses presented here. This methodology is made possible and practical by a confluence of techniques drawn from SMNI itself, modern methods of functional stochastic calculus defining nonlinear Lagrangians, VFSR, and parallel-processing computation.

It should not be too surprising that our present project is at a similar stage to where we began: The issues presented in modeling and understanding combat are quite similar, mathematically and operationally, to the issues presented in modeling and understanding the human brain! 


\section{References}

${ }^{1}$ Bartlett, G.T., "Battle Command Training Program," Phalanx, Vol. 21, 1988, pp. 18-20.

2 Comptroller General, "Models, Data, and War: A Critique of the Foundation for Defense Analyses," U.S. General Accounting Office, Washington, DC, Report No. PAD-80-21, 1980.

${ }^{3}$ Comptroller General, "DOD Simulations: Improved Assessment Procedures Would Increase the Credibility of Results," U.S. General Accounting Office, Washington, DC, Report No. GAO/PEMD-88-3, 1987.

${ }_{5}^{4}$ Jammer, M., The Philosophy of Quantum Mechanics, Wiley \& Sons, New York, NY, 1974.

${ }^{5}$ Ingber, L., The Karate Instructor's Handbook, Physical Studies Institute-Institute for the Study of Attention, Solana Beach, CA, 1976.

${ }_{7}^{6}$ Ingber, L., Karate: Kinematics and Dynamics, Unique, Hollywood, CA, 1981.

${ }^{7}$ Ingber, L., Elements of Advanced Karate, Ohara, Burbank, CA, 1985.

8 Ingber, L., "Editorial: Learning to learn," Explore, Vol. 7, 1972, pp. 5-8.

${ }_{10}^{9}$ Ingber, L., "Attention, physics and teaching," J. Social Biol. Struct., Vol. 4, 1981, pp. 225-235.

${ }^{10}$ van Creveld, M., Command in War, Harvard University Press, Cambridge, MA, 1985.

11 Bowman, M., "Integration of the NTC Tactical Database and JANUS(T) Towards a Combat Decision Support System,” Naval Postgraduate School, Monterey, CA, M.S. Thesis, 1989.

${ }^{12}$ Ingber, L., "Mathematical comparison of JANUS(T) simulation to National Training Center," The Science of Command and Control: Part II, Coping With Complexity, edited by S.E. Johnson and A.H. Levis, AFCEA International, Washington, DC, 1989, pp. 165-176.

13 Dupuy, T.N., "Can we rely on computer combat simulations?," Armed Forces J., Vol. August, 1987, pp. 58-63.

${ }^{14}$ Dockery, J.T. and Santoro, R.T., "Lanchester revisited: Progress in modeling $C^{2}$ in combat," Signal, Vol. July, 1988, pp. 41-48.

${ }^{15}$ Ingber, L., Fujio, H., and Wehner, M.F., "Mathematical comparison of combat computer models to exercise data," Mathl. Comput. Modelling, Vol. 15, Series 1, 1991, pp. 65-90.

${ }^{16}$ Ingber, L., "Mathematical comparison of computer models to exercise data," Symposium on Data Efficiency Using Pre-Processing, edited by T.K. Gardenier, TKG Consultants, Vienna, VA, 1989, pp. 72-115.

${ }^{17}$ Dekker, H., "Quantization in curved spaces," Functional Integration: Theory and Applications, edited by J.P. Antoine and E. Tirapegui, Plenum, New York, 1980, pp. 207-224.

${ }^{18}$ Grabert, H. and Green, M.S., "Fluctuations and nonlinear irreversible processes," Phys. Rev. A, Vol. 19, 1979, pp. 1747-1756.

${ }^{19}$ Graham, R., "Covariant formulation of non-equilibrium statistical thermodynamics," Z. Physik, Vol. B26, 1977, pp. 397-405.

${ }^{20}$ Graham, R., "Lagrangian for diffusion in curved phase space," Phys. Rev. Lett., Vol. 38, 1977, pp. 51-53.

21 Langouche, F., Roekaerts, D., and Tirapegui, E., Functional Integration and Semiclassical Expansions, Reidel, Dordrecht, The Netherlands, 1982.

${ }^{22}$ Schulman, L.S., Techniques and Applications of Path Integration, J. Wiley \& Sons, New York, 1981.

23 Ingber, L., "Nonlinear nonequilibrium statistical mechanics approach to $\mathrm{C}^{3}$ systems," 9th MIT/ONR Workshop on $C^{3}$ Systems: Naval Postgraduate School, Monterey, CA, 2-5 June 1986, MIT, Cambridge, MA, 1986, pp. 237-244.

${ }^{24}$ Fox, R.F., "Uniform convergence to an effective Fokker-Planck equation for weakly colored noise," Phys. Rev. A, Vol. 34, 1986, pp. 4525-4527.

${ }^{25}$ van der Broeck, C., "On the relation between white shot noise, Gaussian white noise, and the dichotomic Markov process," J. Stat. Phys., Vol. 31, 1983, pp. 467-483.

${ }^{26}$ Ingber, L., "Towards a unified brain theory," J. Social Biol. Struct., Vol. 4, 1981, pp. 211-224.

${ }^{27}$ Ingber, L., "Statistical mechanics of neocortical interactions. I. Basic formulation," Physica D, Vol. 5, 1982, pp. 83-107.

${ }_{28}$ Ingber, L., "Statistical mechanics of neocortical interactions. Dynamics of synaptic modification," Phys. Rev. A, Vol. 28, 1983, pp. 395-416. 
${ }^{29}$ Ingber, L., "Statistical mechanics of neocortical interactions. Derivation of short-term-memory capacity," Phys. Rev. A, Vol. 29, 1984, pp. 3346-3358.

"Ingber, L., "Statistical mechanics of neocortical interactions. EEG dispersion relations," IEEE Trans. Biomed. Eng., Vol. 32, 1985, pp. 91-94.

${ }^{31}$ Ingber, L., "Statistical mechanics of neocortical interactions: Stability and duration of the $7 \pm 2$ rule of short-term-memory capacity," Phys. Rev. A, Vol. 31, 1985, pp. 1183-1186.

32 Ingber, L., "Towards clinical applications of statistical mechanics of neocortical interactions," Innov. Tech. Biol. Med., Vol. 6, 1985, pp. 753-758.

33 Ingber, L., "Statistical mechanics of neocortical interactions," Bull. Am. Phys. Soc., Vol. 31, 1986, pp. 868.

${ }^{34}$ Ingber, L., "Applications of biological intelligence to Command, Control and Communications," Computer Simulation in Brain Science: Proceedings, University of Copenhagen, 20-22 August 1986, edited by R. Cotterill, Cambridge University Press, London, 1988, pp. 513-533.

${ }^{35}$ Ingber, L., "Statistical mechanics of mesoscales in neocortex and in command, control and communications $\left(C^{3}\right)$ : Proceedings, Sixth International Conference, St. Louis, MO, 4-7 August 1987," Mathl. Comput. Modelling, Vol. 11, 1988, pp. 457-463.

36 Ingber, L., "Mesoscales in neocortex and in command, control and communications $\left(\mathrm{C}^{3}\right)$ systems," Systems with Learning and Memory Abilities: Proceedings, University of Paris 15-19 June 1987, edited by J. Delacour and J.C.S. Levy, Elsevier, Amsterdam, 1988, pp. 387-409.

${ }^{37}$ Ingber, L. and Nunez, P.L., "Multiple scales of statistical physics of neocortex: Application to electroencephalography," Mathl. Comput. Modelling, Vol. 13, Series 7, 1990, pp. 83-95.

${ }^{38}$ Ingber, L., "Statistical mechanics of neocortical interactions: A scaling paradigm applied to electroencephalography," Phys. Rev. A, Vol. 44, Series 6, 1991, pp. 4017-4060.

39 Ingber, L., "Generic mesoscopic neural networks based on statistical mechanics of neocortical interactions," Phys. Rev. A, Vol. 45, Series 4, 1992, pp. R2183-R2186.

${ }^{40}$ Ingber, L., "Statistical mechanics of multiple scales of neocortical interactions," Neocortical Dynamics and Human EEG Rhythms, edited by P.L. Nunez, Oxford University Press, New York, NY, 1995, pp. 628-681.

${ }^{4}$ Ingber, L., "Path-integral Riemannian contributions to nuclear Schrödinger equation," Phys. Rev. D, Vol. 29, 1984, pp. 1171-1174.

${ }^{42}$ Ingber, L., "Riemannian contributions to short-ranged velocity-dependent nucleon-nucleon interactions," Phys. Rev. D, Vol. 33, 1986, pp. 3781-3784.

${ }^{43}$ Ingber, L., "Statistical mechanics of nonlinear nonequilibrium financial markets," Math. Modelling, Vol. 5, Series 6, 1984, pp. 343-361.

${ }^{44}$ Ingber, L., "Statistical mechanical aids to calculating term structure models," Phys. Rev. A, Vol. 42, Series 12, 1990, pp. 7057-7064.

${ }^{45}$ Ingber, L., Wehner, M.F., Jabbour, G.M., and Barnhill, T.M., "Application of statistical mechanics methodology to term-structure bond-pricing models," Mathl. Comput. Modelling, Vol. 15, Series 11, 1991, pp. 77-98.

46 Ingber, L., "Mathematical comparison of computer models to exercise data," $1989 \mathrm{JDL} \mathrm{C}^{2}$ Symposium: National Defense University, Washington, DC, 27-29 June 1989, SAIC, McLean, VA, 1989, pp. 169-192.

47 Ingber, L. and Sworder, D.D., "Statistical mechanics of combat with human factors," Mathl. Comput. Modelling, Vol. 15, Series 11, 1991, pp. 99-127.

${ }^{48}$ Ingber, L., "Statistical mechanical measures of performance of combat," Proceedings of the 1991 Summer Computer Simulation Conference 22-24 July 1991, Baltimore, MD, edited by D. Pace, Society for Computer Simulation, San Diego, CA, 1991, pp. 940-945. 39-63.

${ }^{49}$ Zadeh, L., "A computational theory of dispositions," Int. J. Intelligent Sys., Vol. 2, 1987, pp.

${ }^{50}$ Goodman, I.R., "A probabilistic/possibilistic approach to modeling $\mathrm{C}^{3}$ systems part II," 1987 Symposium on $C^{3}$ Research at National Defense University, Washington, DC, National Defense University, Washington, DC, 1988, pp. 41-48.

${ }^{51}$ Goldsmith, M. and Hodges, J., "Applying the National Training Center experience: Tactical reconnaissance,” RAND, Santa Monica, CA, Report No. N-2628-A, 1987. 
${ }^{52}$ Kishida, K., "Physical Langevin model and the time-series model in systems far from equilibrium," Phys. Rev. A, Vol. 25, 1982, pp. 496-507.

${ }^{53}$ Kishida, K., "Equivalent random force and time-series model in systems far from equilibrium," $J$. Math. Phys., Vol. 25, 1984, pp. 1308-1313.

54 Mathews, J. and Walker, R.L., Mathematical Methods of Physics, 2nd ed., Benjamin, New York, NY, 1970.

55 Ingber, L., "Very fast simulated re-annealing," Mathl. Comput. Modelling, Vol. 12, Series 8, 1989, pp. 967-973. $157-162$. Szu, H. and Hartley, R., "Fast simulated annealing," Phys. Lett. A, Vol. 122, Series 3-4, 1987, pp.

${ }^{57}$ Kirkpatrick, S., Gelatt, C.D., Jr., and Vecchi, M.P., "Optimization by simulated annealing," Science, Vol. 220, Series 4598, 1983, pp. 671-680.

${ }^{58}$ Ingber, L. and Rosen, B., "Genetic algorithms and very fast simulated reannealing: A comparison," Mathl. Comput. Modelling, Vol. 16, Series 11, 1992, pp. 87-100.

59 Ingber, L., "Simulated annealing: Practice versus theory," Mathl. Comput. Modelling, Vol. 18, Series 11, 1993, pp. 29-57.

${ }^{60}$ Rosen, B., "Function optimization based on advanced simulated annealing," IEEE Workshop on Physics and Computation - PhysComp '92, , 1992, pp. 289-293.

${ }^{61}$ Ingber, L. and Rosen, B., "Very Fast Simulated Reannealing (VFSR)," University of Texas, San Antonio, TX, [ringer.cs.utsa.edu: /pub/rosen/vfsr.Z], 1992.

${ }^{62}$ Wehner, M.F. and Wolfer, W.G., "Numerical evaluation of path-integral solutions to FokkerPlanck equations. I.," Phys. Rev. A, Vol. 27, 1983, pp. 2663-2670.

${ }^{63}$ Wehner, M.F. and Wolfer, W.G., "Numerical evaluation of path-integral solutions to FokkerPlanck equations. II. Restricted stochastic processes," Phys. Rev. A, Vol. 28, 1983, pp. 3003-3011.

64 Wehner, M.F. and Wolfer, W.G., "Numerical evaluation of path integral solutions to FokkerPlanck equations. III. Time and functionally dependent coefficients," Phys. Rev. A, Vol. 35, 1987, pp. 1795-1801.

65 Theiler, J., "Correlation dimension of filtered noise," UC San Diego, La Jolla, CA, Report 6/29/1988, 1988.

${ }_{67}^{60}$ Pool, R., "Is it chaos, or is it just noise?," Science, Vol. 243, 1989, pp. 25-28.

${ }^{67}$ Rapp, P.E., Albano, A.M., Schmah, T.I., and Farwell, L.A., "Filtered noise can mimic low dimensional chaotic attractors," Phys. Rev. E, Vol. 47, Series 4, 1993, pp. 2289-2297.

${ }^{68}$ Brock, W.A., "Distinguishing random and deterministic systems: Abridged version," J. Econ. Theory, Vol. 40, 1986, pp. 168-195.

${ }_{70}$ Grassberger, P., "Do climatic attractors exist?," Nature, Vol. 323, 1986, pp. 609-612.

70 Abarbanel, H.D.I., Brown, R., and Kadtke, J.B., "Prediction in chaotic nonlinear systems: Methods for time series with broadband Fourier spectra," Phys. Rev. A, Vol. 41, 1990, pp. 1782-1807.

${ }^{71}$ Sworder, D.D. and Clapp, G.A., "Supervisory control of C $C^{3}$ systems," 1989 JDL $C^{2}$ Symposium at National Defense University, Washington, DC, SAIC, McLean, VA, 1989, pp. 478-482.

72 Miller, G.J. and Bonder, S., "Human factors representations for combat models," Defense Tech. Info. Ctr., Alexandria, VA, Vector Research Report AD-A133351, 1982.

${ }^{73}$ Van Nostrand, S., "Including the Soldier in Combat Models," Industrial College of the Armed Forces, Fort McNair, Washington, DC, Thesis S73, 1988.

${ }_{75}^{74}$ Dupuy, T.N., Numbers, Predictions \& War, Bobbs-Merrill, Indianapolis, MN, 1979.

75 Sworder, D.D. and Clapp, G.A., "Quantifying uncertainty in $C^{3}$ decision makers," $1990 \mathrm{JDL} \mathrm{C}^{2}$ Symposium: Naval Postgraduate School, Monterey, CA, June 1990, SAIC, McLean, VA, 1990, pp. 348-353.

${ }^{76}$ Upton, S.C., "A Statistical Mechanics Model of Combat," Naval Postgraduate School, Monterey, CA, M.S. Thesis, 1987.

${ }^{77}$ Gallagher, J.F., “A Joint Army-Navy Combat Model Using TLAM C/D," Naval Postgraduate School, Monterey, CA, M.S. Thesis, 1988.

78 Balaconis, R.J., "Integrated Strike Warfare High Fidelity Simulation: Cruise Missile and TACAIR Support of AirLand Battle," Naval Postgraduate School, Monterey, CA, M.S. Thesis, 1989. 
${ }^{79}$ Connell, J.C., Jr., "Memory Efficient Evaluation of Nonlinear Stochastic Equations and C ${ }^{3}$ Applications," Naval Postgraduate School, Monterey, CA, M.S. Thesis, 1987.

80 Yost, C.P., "A Virtual Statistical Mechanical Neural Computer," Naval Postgraduate School, Monterey, CA, M.S. Thesis, 1987.

${ }^{81}$ Richardson, W.E., Miller, J.R., and Murphy, C.G., "Command, control, and communications $\left(\mathrm{C}^{3}\right)$ technology projection and assessment advanced computer architectures," Defense Communications Agency, Reston, VA, Technical Report, 1988.

82 Pennisi, E., "The new neural network tussle: 'top down' or 'bottom up'," The Scientist, Vol. 2 (21), 1988, pp. 1,4-5. 


\section{Figure Captions}

Fig 1 NTC vs Janus(T), attrition ("kills") data for an NTC mission (upper left box) and for three Janus(T) runs using the NTC qualified database.

Fig 2 Path integral calculation of long-time distribution: The horizontal axes represent red and blue forces. For this Janus(T) additive noise case, two time slices are superimposed. Taking the initial onset of the engagement as 35 minutes, these peaks represent 50 and 100 minutes.

Fig 3 Attrition means: The left two boxes are blue; the right two boxes are red. The left-hand-side box of each pair represents Janus(T); the right-hand-side box represents NTC; solid lines the additive noise model; dotted lines the multiplicative noise model; small circles in the means' boxes empirical data.

Fig 4 Attrition variances.

Fig 5 Integrated strike warfare scenarios; rectangles represent the six scenarios that were wargamed. Fig 6 Ongoing development of joint Janus(T).

Fig 7 Support of Higher-Echelon Models.

Fig 8 Comparison of NTC Models. The first graph shows the NTC additive noise model. The second graph shows the NTC multiplicative noise model with the same view and scale. 
NTC KLLS DAIA - ROIATION

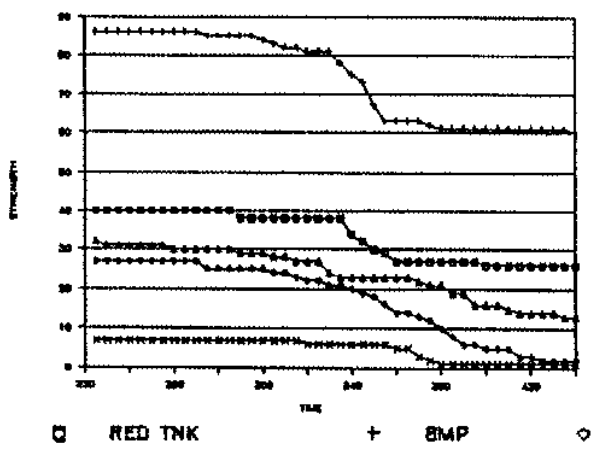

JANUS(T)/NTC RUN A2

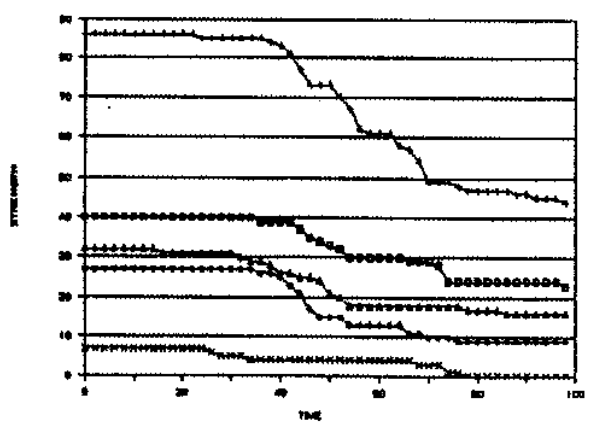

JANUS(T)/NTC RUN A1

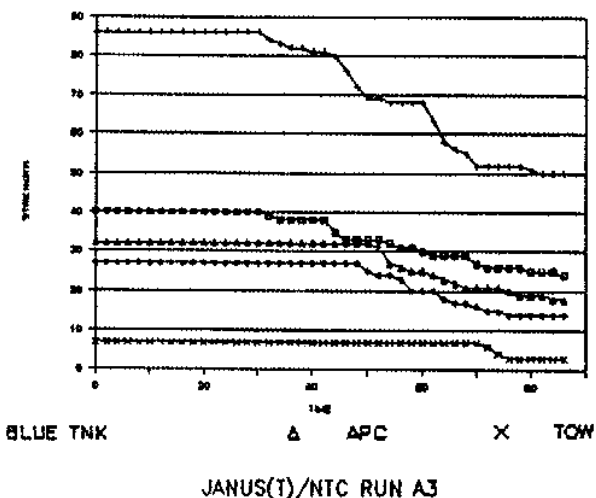

JANUS(T)/NTC RUN A3

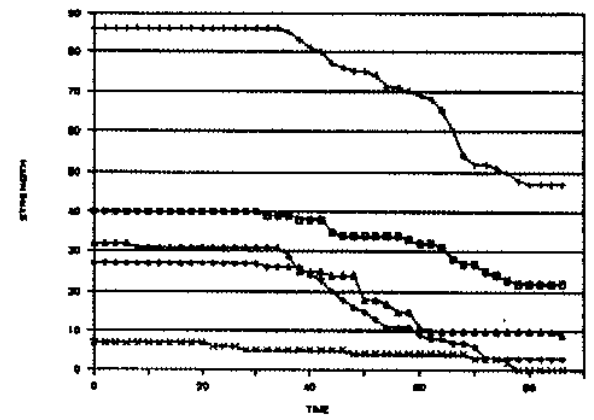




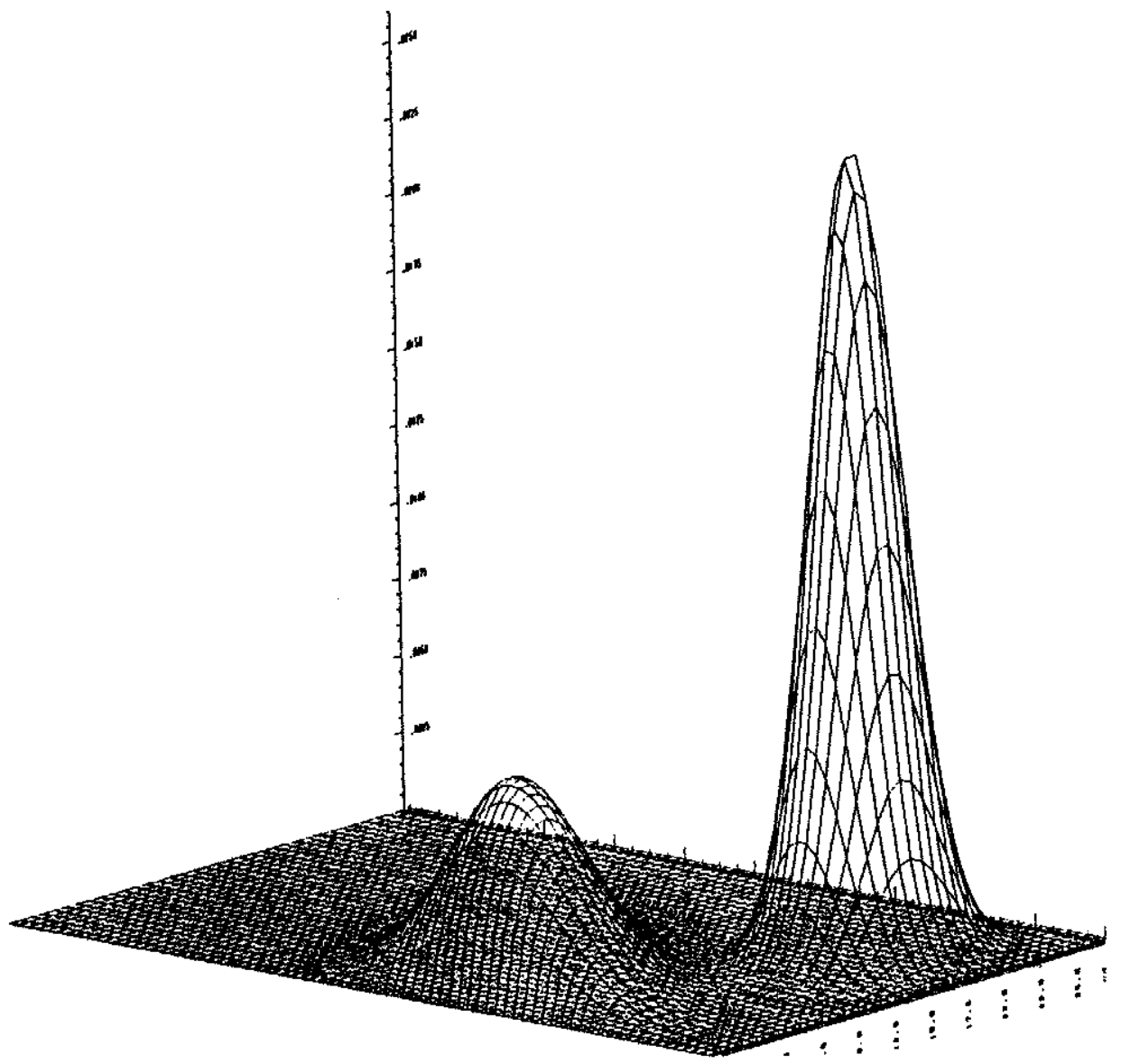


JANUS(T) \& NTC Attrition Means
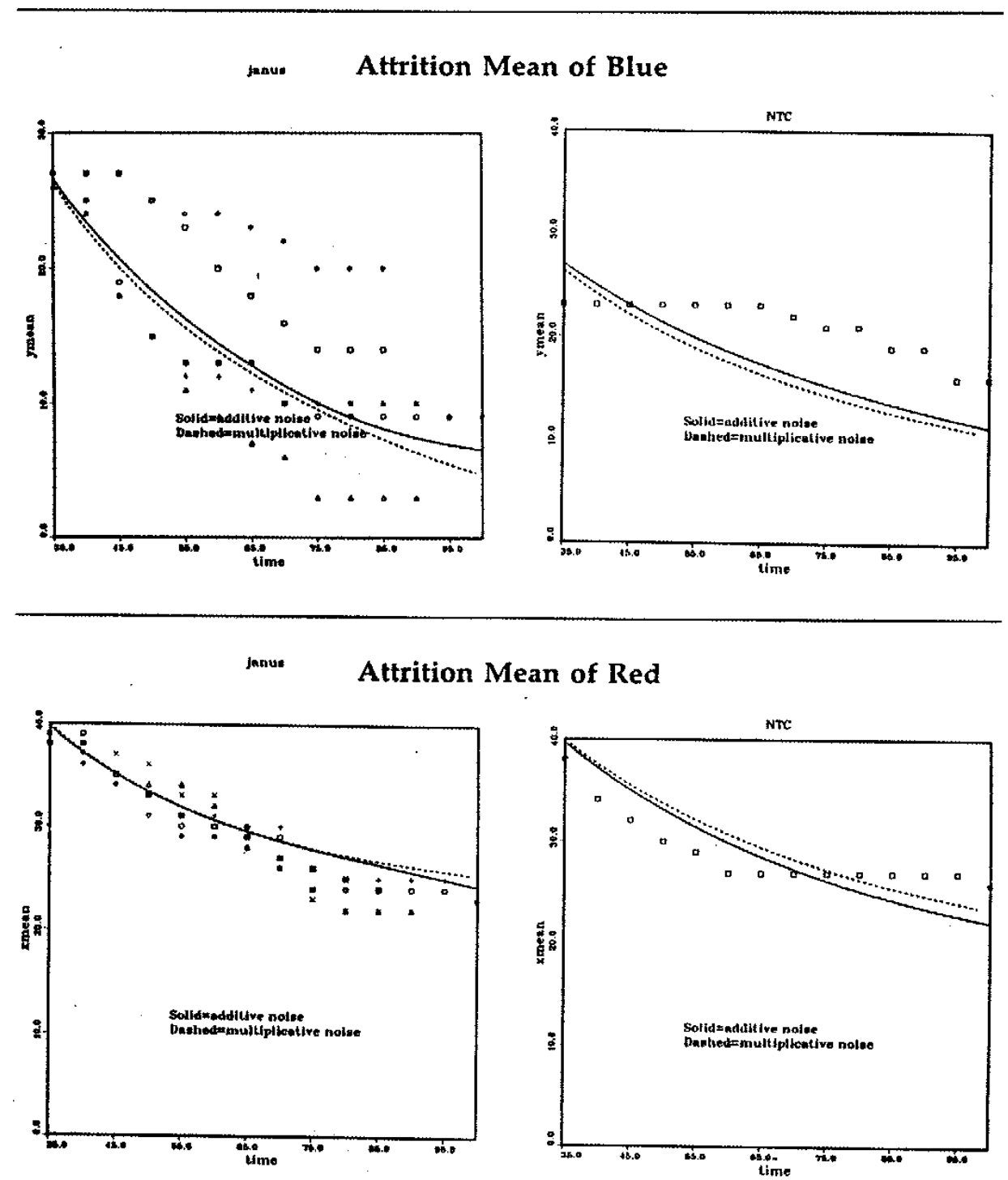
JANUS(T) \& NTC Attrition Variances

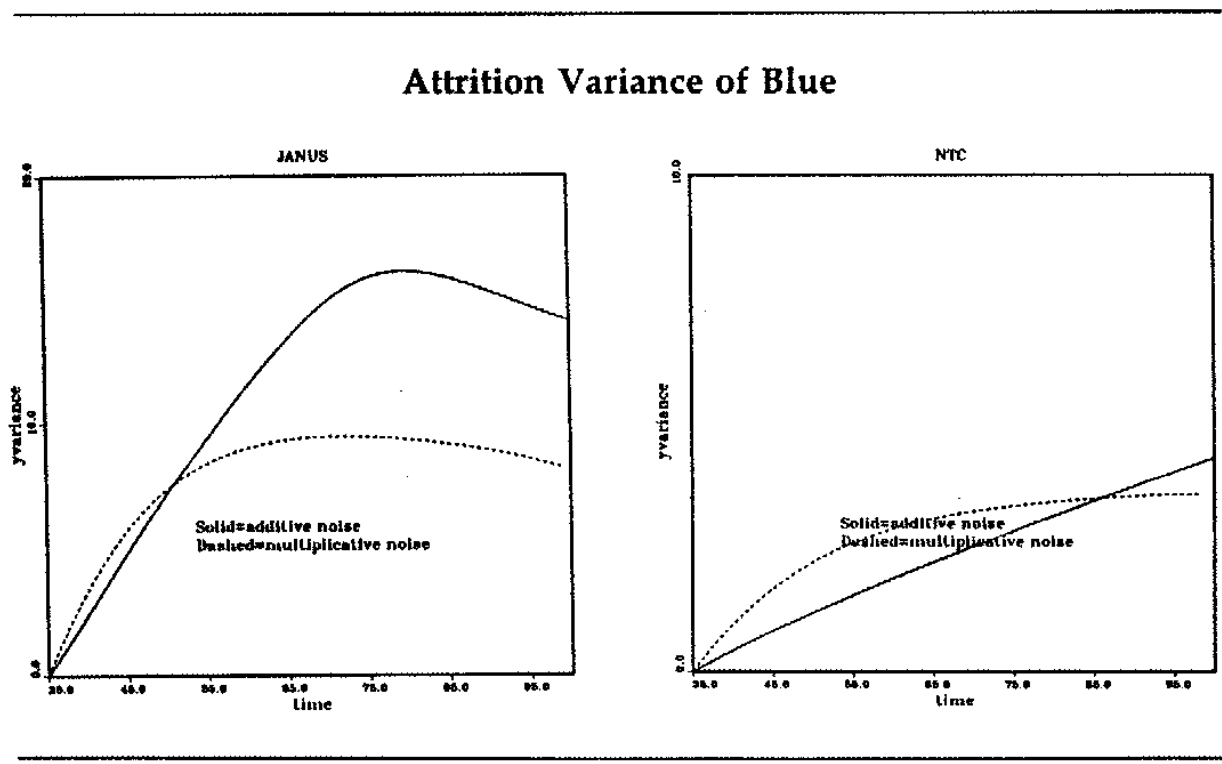

Attrition Variance of Red
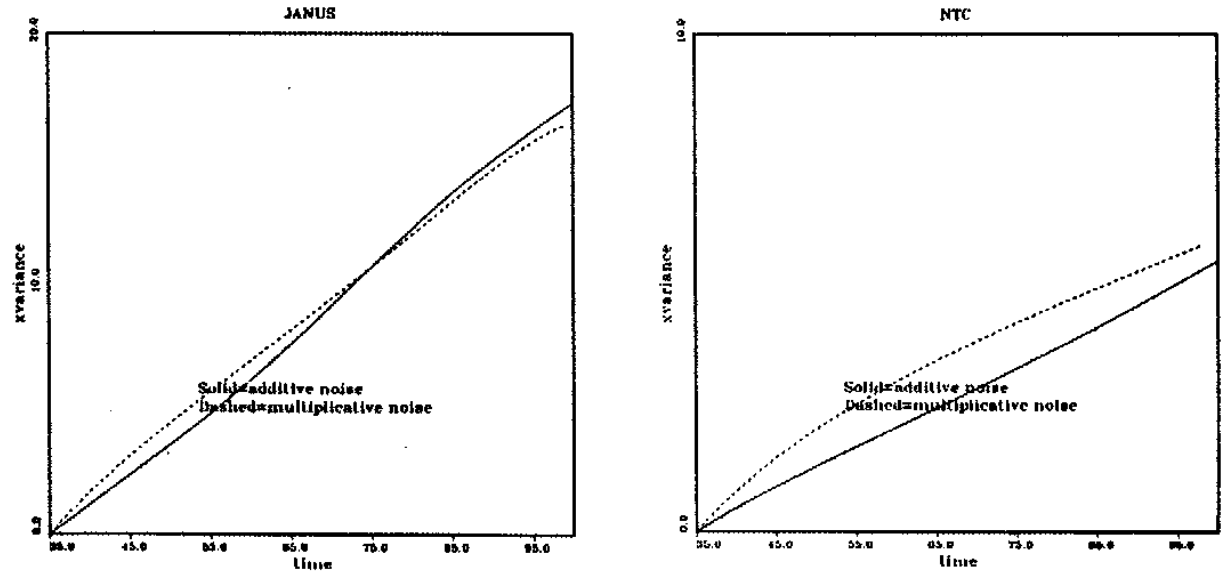


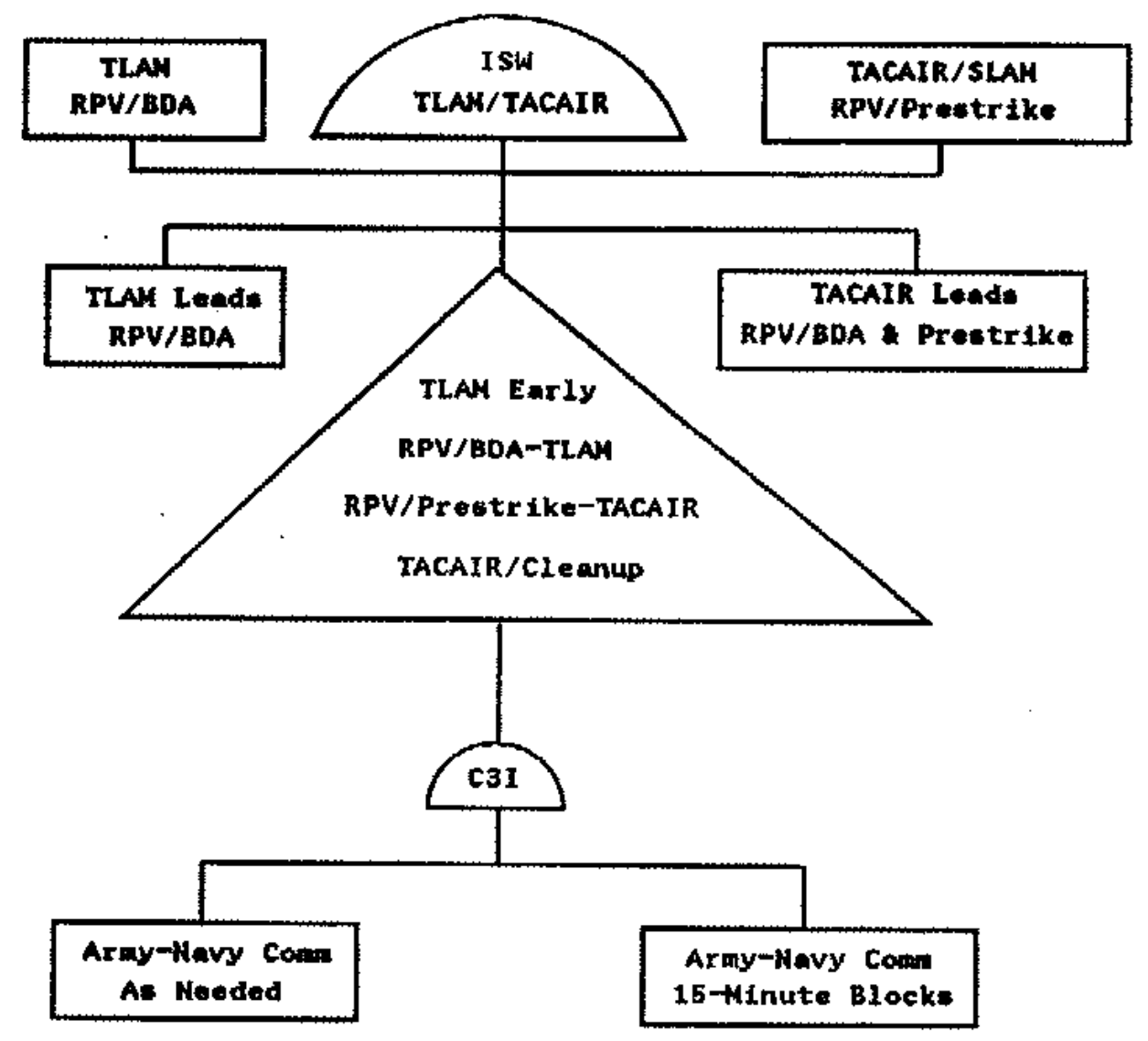




\begin{tabular}{|c|c|c|c|}
\hline $\begin{array}{l}\text { Increase Air-to- } \\
\text { Ground weapons capa- } \\
\text { bilities of Strike } \\
\text { Alrcraft to Include } \\
\text { TACIT RAINBOW" } \\
\text { and "HARM" antl- } \\
\text { radiation weapons }\end{array}$ & $\begin{array}{l}\text { Increase "ajr" dimen- } \\
\text { sion in the AirLand } \\
\text { scenario developinent; } \\
\text { lntroduce F-14 naval } \\
\text { figlerter aircraft. Build } \\
\text { on "air defense" system } \\
\text { on " "nyer" with direct } \\
\text { lire weapon sy'stems. }\end{array}$ & $\begin{array}{c}\text { Introduce Alr Force } \\
\text { F-15, F-16, and ATF } \\
\text { expanding the } \\
\text { jointness" and usage } \\
\text { of joint-JANUS (T) } \\
\text { model. }\end{array}$ & $\begin{array}{l}\text { Expand naval usage by } \\
\text { modelling more anti- } \\
\text { surface weapon sys- } \\
\text { tems (i.e., TASM, } \\
\text { Harpoon) }\end{array}$ \\
\hline $\begin{array}{c}\text { Aircran Carrier Battic } \\
\text { Group (CVBG) with } \\
\text { AEGIS projecting In- } \\
\text { tegrated Strike War- } \\
\text { fare }\end{array}$ & $\begin{array}{l}\text { U.S. Navy Altack Air- } \\
\text { craft }(\text { F/A-18 and } A-6)\end{array}$ & $\begin{array}{l}\text { Introduction of the } \\
\text { Standof Land Attack } \\
\text { Missile (SLAM) } \\
\text { RPVs, and TLAM-D } \\
\text { (GPS) }\end{array}$ & $\begin{array}{l}\text { Coastal/Near Coast } \\
\text { Scenario on NATO's } \\
\text { Central Front (North- } \\
\text { em Flank) }\end{array}$ \\
\hline \multicolumn{4}{|c|}{ Development of TLAM-C Weapon System with the BBBG in a Persian Gulf Scenario } \\
\hline \multicolumn{4}{|c|}{$\begin{array}{l}\text { Validation of JANUS (T) Through Mathentatical Comparison of JANLS (T) to National Training } \\
\text { Center Data. Establish Credibility Bascd On Correspondence to the real (exercise) world }\end{array}$} \\
\hline
\end{tabular}




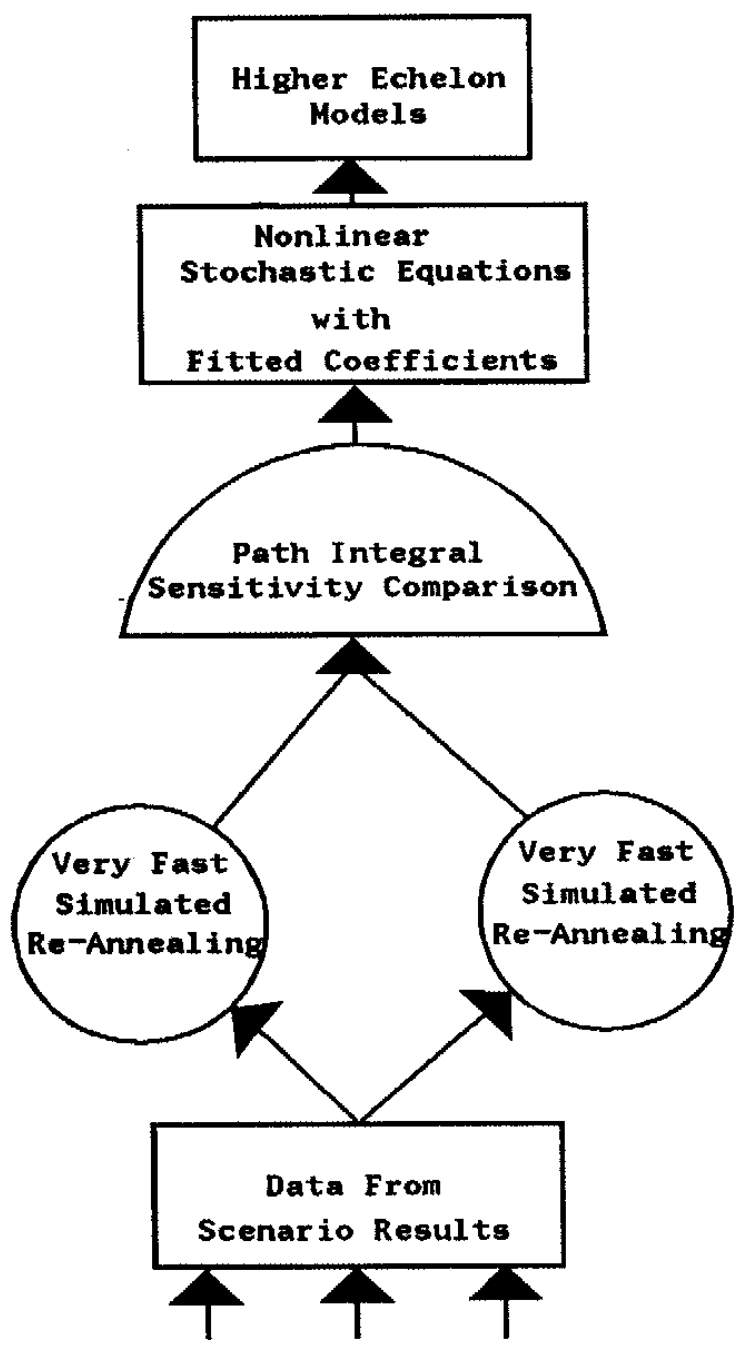


NTC Additive Noise Model

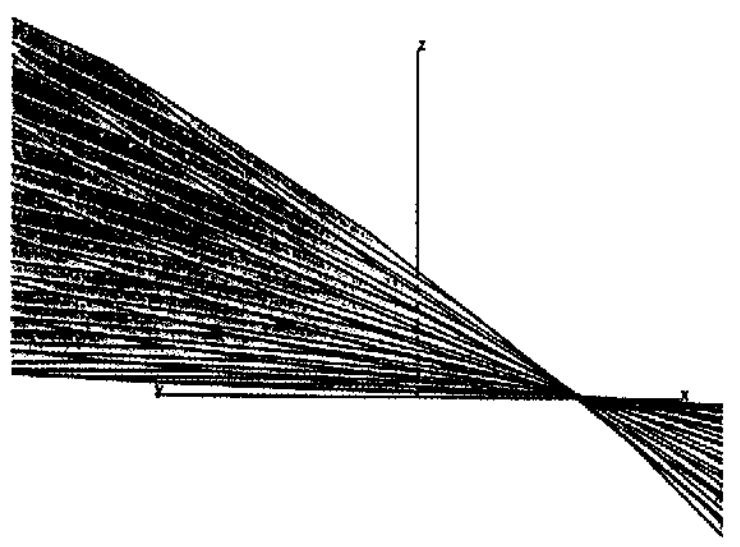

NrC Multiplicative Noise Model

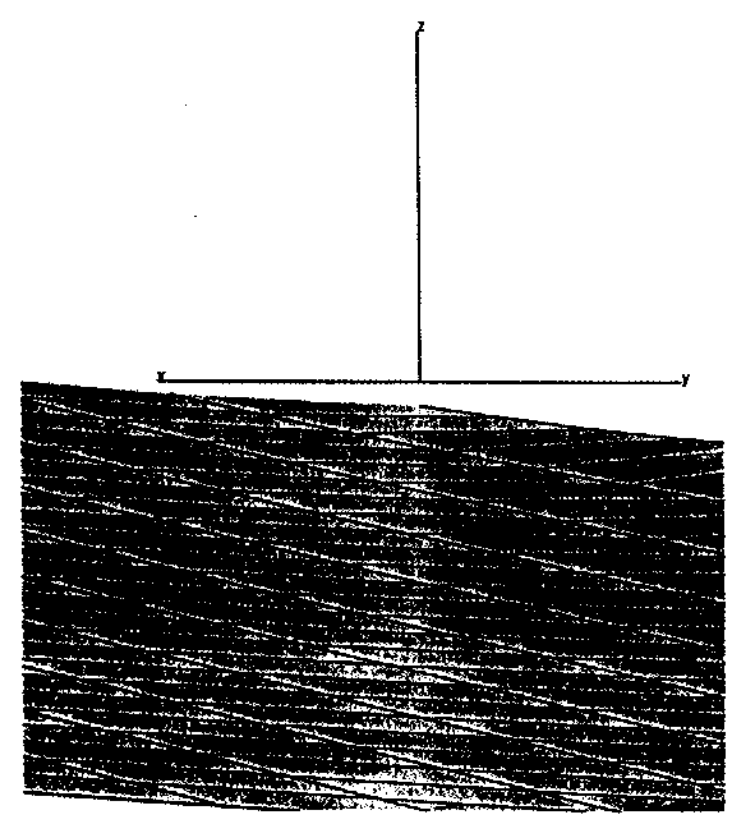

